Proyecioness

Vol. 15, $N^{\circ} 1$, pp. $47-90$, July $1996 \quad$ DOl: $10.22199 / 507160917.1996 .0001 .00004$ Universidad Católica del Norte

Antofagasta - (hile

\title{
CLOSED RIEMANN SURFACES WITH DIHEDRAL GROUPS OF CONFORMAL AUTOMORPHISMS
}

\author{
Rubén A. Hidalgo* \\ Universidad Técnica Federico Santa Maria, Valparaiso, Chile.
}

\begin{abstract}
In these notes we show that if $H$ is a group of conformal automorphisms, isomorphic to a dihedral group, acting free fixed points on a closed Riemann surface $S$, then there is a Schottky uniformization of $S$ for which $H$ lifts. We also give an explicit example of a dihedral group for which the above lifting property fails, shouring in this way that condition (A) of [4] is not sufficient in general.
\end{abstract}

"This work was partially supported by projects Fondecyt 1950830 and UTFSM 951221 


\section{Introduction}

In these notes we obtain some results in the theory of uniformization of closed Riemann surfaces with automorphisms. In [4] we were interested in the following question. Let us consider a closed Riemamn surface $S$ and a group $H$ of conformal automorphisms of it. Is there some Schottky covering $(G, \Omega, \pi: \Omega \rightarrow S)$ for which $H$ lifts? (that is, for each $h \in H$ there is a $\tilde{h} \in \operatorname{Ant}(\Omega)$ satisfying $h \pi=\pi \tilde{h})$.

In [10] L. Keen solves this problem for $S$ hyperelliptic and $H$ the gromp generated by the hyperelliptic involution. In [5] we solve this problem for pairs $(S, H)$, where $H$ is any cyclic group of order two.

In [4] we obtain necessary conditions to be satisfied by $H$ in order to find a Schottky covering as desired. These necessary conditions only concern to the set of fixed points of non-trivial elements of $H$. If $H$ is isomorphic to a dihedral group, then these conditions hold trivially (see section 5).

In [6], [7] and [8] we show that these conditions are sufficient for $H$ isomorphic either to $\mathbf{Z}, \mathbf{Z} / 2 \mathbf{Z} \oplus \mathbf{Z} / 2 \mathbf{Z}$ and abelian in general, respectively.

The aim of these notes is to study this problem for dihedral groups of conformal automorphisms. If the action of the group is free fixed points, then we show the existence of a Schottky covering of the surface $S$ for which the group $H$ lifts. We also describe in full the situation when the genus of $S$ is one (see section 4 ).

Since the dihedral group of order four is abelian, it follows from the results in [8] that we are able to find a Schottky covering for which the group lifts.

The first hope is that for a general dihedral group the above result is true. Unfortunately, this is not the case as shown in the example given in section 8 . This example is a dihedral group of order six acting on a surface of genus four with, by Riemann-Hurwitz, fixed points. In section 7 we show that in the absence of fixed points, we may find a Schottky covering as desired. We give a list, in section 9, of geometrically finite Kleinian groups $J$, containing a Schottky group $G$ as a normal subgroup of finite index, with $J / G$ isomorphic to a dihedral group. Such a list gives a lot of different topological actions of a dihedral group on a closed Riemamn surface. We expect such a list to be complete, that is, all diliedral actions that satisfy the lifting problem are represented in it.

As a consequence of the Nielsen realization theorem [11], the above describes all free fixed points topological actions of a dilhedral group of orientation preserving homeomorphisms on a closed orientable surface.

The development of these notes is as follows. 
Section 1: Definition of Schottky coverings, and recall of a well known fac:t about conformal automorphisms of region of discontinuity of Schot tky groups.

Section 2: Some basics from quasiconformal deformation theory.

Section 3: The lifting problem and the main theorem. We also state the main theorem from the points of view of group theory and of 3-manifolds.

Section 4: The proof of the main theorem for Riemann surfaces of gems zero and one.

Section 5: Information about the fixed points of the elements of a clihedral group of conformal automorphisms on a closed Riemann surfact.

Section 6: The case when the quotient Riemann surface has genus greater or equal to one. Construction of a special set of oriented simple loops.

Section 7: Proof of the main theorem.

Section 8: A counterexample for the lifting problem for dihedral groups acting with fixed points.

Section 9: A list of geometrically finite Kleinian groups containing a Schottky group as a normal subgroup of finite index and quotient a dihedral group.

\section{Schottky Coverings}

2.1. Schottky Groups. For $\mathrm{g} \geq 1$, let $C_{k}, C_{k}^{\prime}, k=1, \ldots, g$, be $2 g$ Jordan curves on the Riemann sphere, $\hat{\mathbf{C}}=\mathbf{C} \bigcup\{\infty\}$. Assume that they are mutually disjoint and bound a $2 g$-connected region $D$. Suppose that for each $k$ there exists a Möbius transformation $A_{k}$ with the following properties.

(i) $A_{k}\left(C_{k}\right)=C_{k}^{\prime \prime}$.

(ii) $A_{k}(D) \cap D=\emptyset$.

The group $G$ generated by the transformations $A_{1}, \ldots, A_{g}$ is called a Schottky group of genus $g$.

It is a well known fact $G$ is a purely loxodromic Kleinian group isomorphic to a free group of rank $g$ (see [12] and [13]). A fundamental domain $F$ for $G$ is given by $D$ union the curves $C_{k}, k=1, \ldots, g$. The domain $F$ is called a standard fundamental domain for $G$ with respect to the above generators. This domain is not uniquely determined by the above generators.

In fact, every purely loxodromic Kleinian group isomorphic to a free group of rank $g$ is necessarily a Schottky group of genus $g$ (see [1:3]). 
For our purposes, we define the Schottky group of genus zero to be the group with the identity as its only element, that is, the trivial group.

2.2. The Riemam Surface Associated to a Schottky Group. Let $G$ be a Schottky group of genus $g$, and $\Omega$ its region of discontinuity, then $S=\Omega / G$ is a closed Riemann surface of genus $g$. Moreover, if $A_{1}, \ldots$, $A_{g}$ form a set of free generators for $G$, and $F$ is a standard fundamental domain for these generators, with boundary curves $C_{k}, C^{\prime \prime}{ }_{k}(k=1, \ldots, g)$, then these loops projects to a set of $g$ disjoint homologically independent simple loops on $S$. Reciprocally, the retrosection theorem [2] asserts that we can reverse this situation. Another important fact about Schottky groups is the following.

Theorem 1.1. If $\Omega$ is the region of discontinuity of a Schottky group $G$, then any conformal automorphism of $\Omega$ is the restriction of a Möbius transformation.

2.3. Schottky Coverings. Given a Schottky group $C$, with region of discontinuity $\Omega$, we can associated to it a natural triple $(G, \Omega, \pi: \Omega \rightarrow$ $\Omega / G)$. The holomorphic projection $\pi: \Omega \rightarrow \Omega / G$ is the natural one. We can also get a lot of triple associated to $G$ as $\left(G, \Omega, p: \Omega \rightarrow \Omega / G^{\prime}\right)$, where $p=h \pi t$, the map $t$ is a Möbius transformation that conjugates $G$ into itself and $h$ is an automorphism of the surface $\Omega / G$.

Any triple $(G, \Omega, p: \Omega \rightarrow S)$, where $S$ is a closed Riemamn surface, $G$ a Schottky group with region of discontinuity $\Omega$, and $p: \Omega \rightarrow S$ a holomorphic covering with $G$ as covering group, will be called a Schottky covering of $S$.

\section{Quasiconformal maps}

In this section we recall some basics from the theory of quasiconformal maps (see [1] for more details).

3.1. Quasiconformal homeomorphisms. Let $\mu(z)$ be a measurable function defined on the Riemann sphere with $\|\mu\|_{\infty}<1$. Let $\Phi$ be a orientation preserving homeomorphism of the Riemann sphere. We say that $\Phi$ is $\mu$-quasiconformal homeomorphism if

$$
\partial \Phi / \dot{\partial} \bar{z}=\mu(z) \dot{\partial} \Phi / \dot{\partial} z, \text { a.e. }
$$


In general, an orientation preserving homeomorphism of the Rienann sphere is called quasiconformal if it is $\mu$-quasiconformal for some $\mu$.

Theorem 2.1 (Ahlfors-Bers Theorem [1])。If $\mu(z)$ is a measurable function on the Riemann sphere with $\|\mu\|_{\infty}<1$. then there exists a unique quasiconformal homeomorphism. $W^{\mu}$ of the Riemann sphere salisfying the equation

$$
\dot{\partial} W^{\mu} / \partial \bar{z}=\mu(z) \partial W^{\mu} / \partial z, a . \epsilon^{2},
$$

and $W^{\mu}(0)=0, W^{\mu}(1)=1, W^{\mu}(\infty)=\infty$. Moreover. the solutions $W^{t \mu}$ vary analytically on the complex paraineter $t$.

Lemma 2.2. Let $G$ be a finitely generated Kleinian group and let $\triangle$ be an invariant set of components of the region of discontinuity of $(r$. Suppose? there exists $\mu$ a measurable function on $\triangle$ so that

$$
\mu(g(z)) g^{\prime}(z)=\mu(z) g^{\prime}(z), \text { for all } g \text { in } G \text { and all } z \text { in } \triangle \text {. }
$$

Extend $\mu$ to be zero in the complement of $\triangle$ and let $\Phi$ be a $\mu$-quasiconfor homeomorphism of the Riemann sphere. Then $\Phi g \Phi^{-1}$ is again a Möbius transformation.

The proof of the above lemma is a direct computation and it is left to the interested reader.

3.2. Beltrami coefficients. If $\|\mu\|_{\infty}<1$ in the lemma above, then $\mu$ is called a Beltrami coefficient for the group $G$ supported in $\triangle$.

Let $\mu$ be a Beltrami coefficient for a Kleinian group $C$, and let $\Phi$ be a $\mu$-quasiconformal homeomorphism. Set $\tilde{G}=\Phi G_{x}^{-1}$. Then the group $\tilde{G}^{\prime}$ is again a Kleinian group for which $\Phi(\triangle)$ is invariant set for $\tilde{G}$ in its region of discontinuity $\Phi(\Omega(G))$, where $\Omega(G)$ is the region of discontinuity of $C^{\prime}$. We say that $\tilde{G}$ is obtained by quasiconformal deformation of $G$ on $\triangle$. If the set $\triangle$ is all the region of discontinuity, we only say that $\tilde{G}$ is obtained by a quasiconformal deformation of $G$.

\section{The Main Theorem}

Let $S$ be a closed Riemann surface of genus $g$ and $H$ a group of conformal automorphisms of $S$ isomorphic to a dihedral group. We are interested in studying the following question concerming conformal automorphisms and Schottky coverings. 


\section{Is there a Schottky covering of $S$ for which $H$ lifts?}

The following give a partial answer to the above question in the dihordral case.

Theorem 3.1. Let $S$ and $H$ be a closed Riemann surface and a group of conformal automorphisms of $S$, respectively. If $H$ is isomorphic to a dihedral group and acts free fixed points. then there is a schotthy covering of $S$ for which $H$ lifts.

The dihedral case of order 4 is done in [7] and [8]. In what follows, we consider only dihedral groups of order greater or equal to 6.

In the group theoretical setting, theorem 3.1. can stated as follows.

Theorem 3.2. Let $K$ be a group isomorphic to the fundamental group of a closed orientable surface of genus $\gamma \geq 2$. Assume that $F$ is a normal subgroup of $K$ such that $K / F$ is isomorphic to a dihedral group. Then there exists a common normal subgroup $N$ of $F$ and $K$ such that $F / N$ is a free group of rank $g$.

Theorem 3.1. can be also described from the language of 3-manifolds. A handlebody $V_{g}$ of genus $g \geq 1$ is the connected sum of $g$ copies of the 3-manifold (with boundary) $D \times S^{1}$, where $D$ is the closed unit disc and $S^{1}$ is the unit circle. The boundary $S_{g}$ of the handlebody $V_{g}$ is a closed orientable surface of genus $g$.

The Nielsen realization theorem [11] asserts that we can give to $S_{g}$ the structure of a Riemann surface for which $H$ acts as group of conformal automorphisms. In particular, we can state the main theorem as:

Theorem 3.3. Let $H$ be a group of orientation preserwing homeomorphisms of the boundary $S_{g}$ of some handlebody $V_{g}$. Assume that $H$ acts free fixed points and is isomorphic to a dihedral group. Then there exists an orientation preserving homeomorphism $f: S_{g} \rightarrow S_{g}$, for which $f H f^{\prime \prime}{ }^{1}$ can be extended as group of orientation preserving homeomorphism of $V_{y}$.

In the case the group acts with fixed points is not in general true the existence of such a Schottky covering, as can be seen from the example in section 8 . 


\section{The Case of Gemus 0 and 1}

In this section, we consider all possible actions (by conformal automorphisms) of a dihedral group $H$ on a Riemann surface $S$ of genus $g \in\{0,1\}$. Assume $H$ to be a group of couformal automorphisms of $S$, isomorphic: to a dihedral group of order $2 n, n \geq 3$. The particular case $n=2$ has been already stated in [8].

5.1. Genus Zero Case. If the genus of $S$ is zero, then $S=$ C. Let $(G=\{I\}, \Omega=\hat{\mathbf{C}}, \pi=I: \mathbf{C} \rightarrow \hat{\mathbf{C}})$ be the trivial Schottky covering of $\mathbf{C}$. In this case the group $H$ lifts trivially. We must remark that in this trivial case, every conformal group $H$ (no necessarily finite) lifts.

5.2. Genus One Case. Assume the genus of $S$ to be one, that is, $S$ is a torus. Let $a$ and $b$ be two elements of $H$ of order $n \geq 3$ and 2, respectively. We have that $H$ is generated by $a$ and $b$ with relations $a^{n}=1, b^{2}=1$ and $(a b)^{2}=1$. There are two possibilities for $b$; either it is an involution with four fixed points (a hyperelliptic involution) or it is an involution without fixed points. The automorphism $a$ has four possibilities:

(i) $n=3$ and the torus is conformally equivalent to the torus $\mathbf{C} /<$ $1, e^{i \pi / 3}>$.

(ii) $n=4$ and the torus is conformally equivalent to the torus $\mathbf{C} /<1, i>$.

(iii) $n=6$ and the torus is conformally equivalent to the torus $\mathbf{C} /<$ $1, e^{i \pi / 3}>$.

(iv) $n \geq 3$ and $a$ (and all its powers) has no fixed points.

Here $\mathbf{C}$ denotes the complex plane and $\langle u, v\rangle$ denotes the group generated by the translation $z \rightarrow z+u$ and the translation $z \rightarrow z+v$.

Since the fixed points of $a^{s}$ are permuted by the automorphism $b$, the number of fixed points of $a^{s}$ must be even. We name these fixed points by $p_{1}, \ldots, p_{k}, q_{1}, \ldots, q_{k}$, so that $b\left(p_{i}\right)=q_{i}$. In particular, the rotation numbers (see section 5) satisfy $\alpha\left(a^{s}, p_{i}\right)=-\alpha\left(a^{s}, q_{i}\right)$, if the order of $a^{s}$ is greater than two. It can be checked, from (i)-(iv), that the only possibility is that $a$ (and all its powers) acts without fixed points and the antomorphism $b$ acts with four fixed points (a hyperelliptic involution).

This action can be seen as follows. Let $\tau$ be any complex number with positive imaginary part. Let $T$ be the torus $\mathbf{C} /\langle 1, \tau\rangle$. Choose a complex number $b$, and integers numbers $1 \leq k_{i} \leq n$, at least one $k_{i}$ relatively prime 
to $n, i=1,2$. Let $B(z)=-z+b$ and $A(z)=z+\left(k_{1}+k_{2} \tau\right) / n$. Then the automorphisms $A$ and $B$ descend to automorphisms $a$ (of order $n$ ) and $b$ (of order 2), respectively, generating a dihedral group (of order $2 n$ ) of conformal automorphisms of $T$.

Now choose any simple loop $r$ on the torus $T$, for example the projection of the path $[0,1]=\{z \in \mathbf{C} ; \operatorname{Im}(z)=0,0<\operatorname{Re}(z)<1\}$. This path define a Schottky covering $(G, \Omega, \pi: \Omega \rightarrow T)$ of $T$. Since the transformation $a$ acts trivially on the fundamental group of $T$ and $b$ acts as the transformation $(n, m) \rightarrow(-n,-m)$, they lift to the above Schottky covering.

As a consequence, we obtain the following easy fact.

Theorem 4.1. Let $H$ be a group of conformal automonphisms on a closed Riemann surface $S$ of genus $g \in\{0,1\}$. If $H$ is isomorphic to a dihedral group of order $2 n$, then there is a Schottky covering of $S$ for which $H$ lifts if and only if either:

(1) $n=2$, or

(2) $n \geq 3$ and the cyclic group of order $n$ acts free fixed points. In this case, necessarily, the involutions are hyperelliptic.

\section{Dihedral groups of conformal automorphisms}

In this section, we consider a dihedral group $H$ of conformal automorphisms of a closed Riemann surface $S$. First we proceed to obtain some information about the fixed points of the elements of $H$ (see proposition 5.1). Secondly, we use Riemann-Hurwitz formula [3] to observe that the number of branched values of order two on the quotient surface $S / H$ is always even (see subsection 5.3. and proposition 5.3).

6.1. Stabilizer and Rotation number. Let $S$ be a Riemamn surface and $H$ be a group of conformal automorphisms of $S$. Then

(1) For each $p \in S$, the stabilizer of $p$ in $H$ is the group $H(p)=\{h \in H$ : $h(p)=p\}$.

(2) Let $h \in H$ and $p \in S$ be such that $h(p)=p$. We can find a local coordinate system $(U, \phi)$ such that $\phi(p)=0$ and $\phi h \phi^{-1}(z)=\epsilon^{i \alpha} z$, for all $z \in \phi(U)$. Moreover, we can assume $\phi(U)=\Delta$, where $\triangle$ denotes the unit disc in the complex plane $\mathbf{C}$. The angle $\alpha=\alpha(h, p)$ is well defined up to a multiple of $2 \pi$, independent of the local coordinate, 
and $\alpha\left(h^{k}, p\right)=k \alpha(h, p)$. We may normalize $\alpha$ by assuming that $-\pi<\alpha \leq \pi$. We call $\alpha=\alpha(h, p)$ the rotation number of $h$ at $p$.

6.2. Fixed points. Let us observe that if $a$ and $b$ elements of $H$ of order $n$ and two, respectively, then $H$ is generated by $a$ and $b$, with only relations

$$
a^{n}=b^{2}=(a b)^{2}=1 \text {. }
$$

A non-trivial element of $H$ has the form $a^{k} b^{s}$, where $k \in\{0,1, \ldots, n-$ $1\}$ and $s \in\{0,1\}$. The elements of the form $a^{k} b$ have order two, and for $n \geq 3$, they are exactly those elements of order two. The RiemannHurwitz formula [3] asserts that every automorphism of order two has an even number of fixed points. For fixed $k, 1 \leq k \leq n-1$, if $x$ is a fixed point of $a^{k}$, then $b(x)$ is also a fixed point of it. This is consequence of the relation $b a^{k} b=a^{-k}$. Since the stabilizer of any point is cyclic, we have $b(x) \neq x$. In particular, the automorphism $a^{k}$ has an even number of fixed points. The above relation shows also that if the order of $a^{k}$ is greater than two, then the rotation number of $a^{k}$ at $x$ and $b(x)$ are opposite in sign, that is, $\alpha\left(a^{k}, x\right)=-\alpha\left(a^{k}, b(x)\right)$. It follows from the above arguments that a dihedral group satisfies the necessary conditions of [4] (it was called the condition (A)). We have the following facts concerning the elements of order two in a dihedral group.

\section{Proposition 5.1.}

(1) If $n$ is odd, then $a^{k} b$ is conjugate to $b$. In particular, the number of fixed points of $a^{k} b$ is the same as the number of fixed points of $b$. If $x$ is a fixed point of $a^{k} b$, then $a^{s}(x)$ is not a fixed point of the same $a^{k} b$, for $1 \leq s \leq n-1$.

(2) If $n$ is even, then $a^{2 k} b$ is conjugate to $b$ and $a^{2 k-1} b$ is conjugate to ab. The elements $a b$ and $b$ are non-conjugate. In particular, the number of fixed points of $a^{2 k} b$ (resp., $\left.a^{2 k-1} b\right)$ is the same as for $b($ resp., $a b)$. Moreover, if $x$ is a fixed point of $a^{r} b$, then $a^{n / 2}(x) \neq x$ is also a fixed point of the same $a^{r} b$.

Proof (1) Write $n=2 r-1$ and let $l=(n-k) r$. Then $a^{l} a^{k} b a^{-l}=$ $a^{2 l+k} b=a^{2(n-k) r+k} b=a^{(n-k)(n+1)+k} b=a^{-k+k} b=b$. This shows the first statement. For the second, assume $x$ is a fixed point of $a^{k} b$ and assume 
there exists $s, 1 \leq s \leq n-1$, such that $a^{s}(x)$ is also a fixed point of the same $a^{k} b$. Since the stabilizer of $x$ and also of $a^{s}(x)$ is the group on two elements generated by $a^{k} b$, we must have $a^{s} a^{k} b a^{-s}=a^{k} b$. But the left side of this equality is $a^{2 s+k} b$, so we obtain the equation $a^{2 s}=1$. This is only true for $2 s=n$. This is a contradiction to the fact that $n$ is odd.

(2) The first statement are consequence of the following. $a^{-k} a^{2 k} b a^{k}=$ $a^{-2 k+2 k} b=b$ and $a^{1-k} a^{2 k-1} b a^{k-1}=a^{2-2 k+2 k-1} b=a b$. For the second statement, observe that $a^{n / 2} a^{k} b a^{-n / 2}=a^{n+k} b=a^{k} b$. To see that $a b$ and $b$ are non-conjugate, observe that if we conjugate $b$ by some $a^{s} b$, then we obtain $a^{2 s} b$. If we conjugate $b$ by $a^{s}$, some $1 \leq s \leq n-1$, we also obtain $a^{2 s} b$. This ends the proof of our lemma.

Notation. If $k \in H$, and $\langle k\rangle$ is the cyclic group generated by $k$ in $H$, then we set

$$
F(k)=\{p \in S ;\langle k\rangle=\{h \in H ; h(p)=p\}\} .
$$

The cardinality of $F(k)$ is denoted by $N(k)$. As a consequence of proposition 5.1 , we have the following.

\section{Corollary 5.2.}

(1) For each $s<n$, dividing $n$, we have $N\left(a^{s}\right)=2 s k_{s}$, for some nonnegative integer $k_{s}$. Moreover, if $a^{s}$ has order greater or equal to three, then its 2 sks fixed points can be arranged as

$$
p_{1, i}^{s}, \ldots, p_{s, i}^{s}, q_{1, i}^{s}, \ldots, q_{s, i}^{s}
$$

where $i=1, \ldots, k_{s}$, such that $b\left(p_{j, i}^{s}\right)=q_{j, i}^{s}$ and $a\left(p_{j, i}^{s}\right)=p_{j+1, i}^{s}(j$ modulo $n)$. In particular, $\left.\alpha\left(a^{s}, p_{j, i}^{s}\right)=-\alpha\left(a^{s}, q_{j, i}^{s}\right) \in\right] 0, \pi[$, for all $j$ and $i$.

(2) If $n$ is odd, $N\left(a^{k} b\right)=2 r_{0}$, for all $k$ and some non-negative integer ro.

(3) If $n$ is even, then $N\left(a^{2 k} b\right)=2 r_{1}$ and $N\left(a^{2 k-1} b\right)=2 r_{2}$, for some non-negative integers $r_{1}$ and $r_{2}$, respectively.

6.3. The signature of the quotient surface $\mathbf{S} / \mathbf{H}$. The signature (see [12]) of the quotient Riemann surface $S / H$, of genus $\gamma$, can be then described as follows.

Let $1=s_{1}<s_{2}<\cdots<s_{t} \leq L$, where $L=(n-1) / 2$ for $n$ odd and $L=n / 2$ for $n$ even. Moreover, assume 
(i) $s_{i}$ divides $n$, and

(ii) if $t$ divides $n$, then $t=s_{i}$, for some $i$.

For each $i \in\{1, \ldots, t\}$, the automorphism $a^{s_{i}}$ is the stabilizer of $2 \cdot s_{i} k_{i}$ different points, for some non-negative integer $k_{i}$. The signature of $S / H$ is:

(1) case $n$ odd.

$$
\left(\gamma, 2 r_{0}+\sum_{i=1}^{t} k_{i} ; 2, \ldots, 2, n / s_{1}, \ldots, n / s_{1}, n / s_{2}, \ldots, n / s_{2}, \ldots n / s_{t}, \ldots, n / s_{t}\right),
$$

where there are $2 r_{0}$ numbers 2 and $k_{i}$ numbers $n / s_{i}$, for all $i$.

$(2)$ case $n$ even.

$$
\left(\gamma, r_{1}+r_{2}+\sum_{i=1}^{t} k_{i} ; 2, \ldots, 2, n / s_{1}, \ldots, n / s_{1}, n / s_{2}, \ldots, n / s_{2}, \ldots, n / s_{t}, \ldots, n / s_{t}\right),
$$

where there are $r_{1}+r_{2}$ numbers 2 and $k_{i}$ numbers $n / s_{i}$, for all $i$.

The Rienrann-Hurwitz formula implies the following equalities in each case.

(1) $g=2 n(\gamma-1)+1+n r_{0}+\sum_{i=1}^{t}\left(n-s_{i}\right) k_{i}$, if $n$ is odd.

(2) $g=2 n(\gamma-1)+1+\left(r_{1}+r_{2}\right) n / 2+\sum_{i=1}^{t}\left(n-s_{i}\right) k_{i}$, if $n$ is even.

As observed above, in the odd case, we have an even number of branch values of order 2 on $S / H$. The following shows that the same holds for the even case.

Proposition 5.3. In the even case, $r_{1}+r_{2}$ is even. Proof Let $H$ be a group of conformal automorphisms of a surface $S$. Assume $H$ to be isomorphic to a dihedral group of order $2 n$ with $n$ even. Let $a$ and $b$ generators of $H$, so that $a$ and $b$ have orders $n$ and 2 , respectively. Denote by $S / H$ the quotient Riemann surface and by $\pi: S \rightarrow S / H$ the natural holomorphic: (branched) covering. On $S / H$ we choose a point $x$, which is assumed not a branch value, and on $S$ we choose a point $z$ such that $\pi(z)=x$. We choose a set of simple loops $\alpha_{1}, \ldots, \alpha_{\gamma}, \beta_{1}, \ldots, \beta_{\gamma}$, on $S$ through $x$ so that, they do not contain branch values, they intersect at their common point $x$, and they are a canonical basis for $\Pi_{1}(S / H, x)$, the fundamental group of $S / H$ based at $x$. Choose disjoint oriented simple loops $\delta_{1}, \ldots, \delta_{r_{1}}, \eta_{1}, \ldots, \eta_{r_{2}}, \theta_{1}, \ldots, \theta_{k},\left(k=k_{1}+\cdots k_{t}\right)$, so that

(1) these simple loops are disjoint from the above $\alpha_{i}$ and $\beta_{j}$ loops; 
(2) each loop bounds a topological disc: containing exactly one branch value;

(3) the loop $\delta_{i}$ bounds a branch value of order two, which is projection of a fixed point of $b$;

(4) the loop $\eta_{i}$ bounds a branch value of order two, which is projection of a fixed point of $a b$;

(5) each loops $\theta_{j}$ bounds branch value that is the projection of a fixed point of a power of $a$;

(6) the orientation of each loop is given so that the branch value they bound is at the left side (when going in the positive direction).

We consider a set of oriented simple paths, all of them disjoint, starting at $x$, and such that

(7) each path connect $x$ with exactly one of the above simple loops (bounding branch values);

(8) these paths are disjoint (with the exception of $x$ ) from the loops $\alpha_{i}$ and $\beta_{j}$.

We may consider these loops to be based at $x$, formed by going through a corresponding path from $x$ to the loop, going around the loop, and then back to $x$ (see figure 1 ). We still denoting these new loops as $\delta_{i}, \eta_{j}$ and $\theta_{l}$, respectively.

Set $X$ the surface $S$ with all fixed point deleted, and $R$ the surface $S / H$ with all branch values deleted. We have that $\pi: X \rightarrow R$ is a regular holonorphic covering with $H$ as covering group. Moreover, $\Pi_{1}(R, x)$ has generators $\alpha_{1}, \ldots, \alpha_{\gamma}, \beta_{1}, \ldots, \beta_{\gamma}, \delta_{1}, \ldots, \delta_{r_{1}}, \eta_{1}, \ldots, \eta_{r_{2}}, \theta_{1}, \ldots, \theta_{k}$, and the only relation is

$$
\prod_{i=1}^{\gamma}\left(\alpha_{i} \beta_{i} \alpha_{i}^{-1} \beta_{i}^{-1}\right) \prod_{j=1}^{r_{1}} \delta_{j} \prod_{s=1}^{r_{2}} \eta_{s} \prod_{l=1}^{k} \theta_{l}=1 .
$$

We have a natural surjective homomorphism $\Phi: \Pi_{1}(R, x) \rightarrow H$ given by lifting loops at $z$. In particular, we have that

(9) $\Phi\left(\alpha_{i} \beta_{i} \alpha_{i}^{-1} \beta_{i}^{-1}\right)=a^{p_{i}}$,

(10) $\Phi\left(\delta_{j}\right)=a^{2 q_{j}} b$, 
(11) $\Phi\left(r_{s}\right)=n^{2 n_{s}-1} b$

(12) $\Phi\left(\theta_{l}\right)=n^{3}$.

In consequence, the relation on the generators of $\Pi_{1}(R, x)$ gives us

$$
1=a^{t} b^{r_{1}}+r_{2}
$$

In particular, $r_{1}+r_{2}$ must be alı even munber.

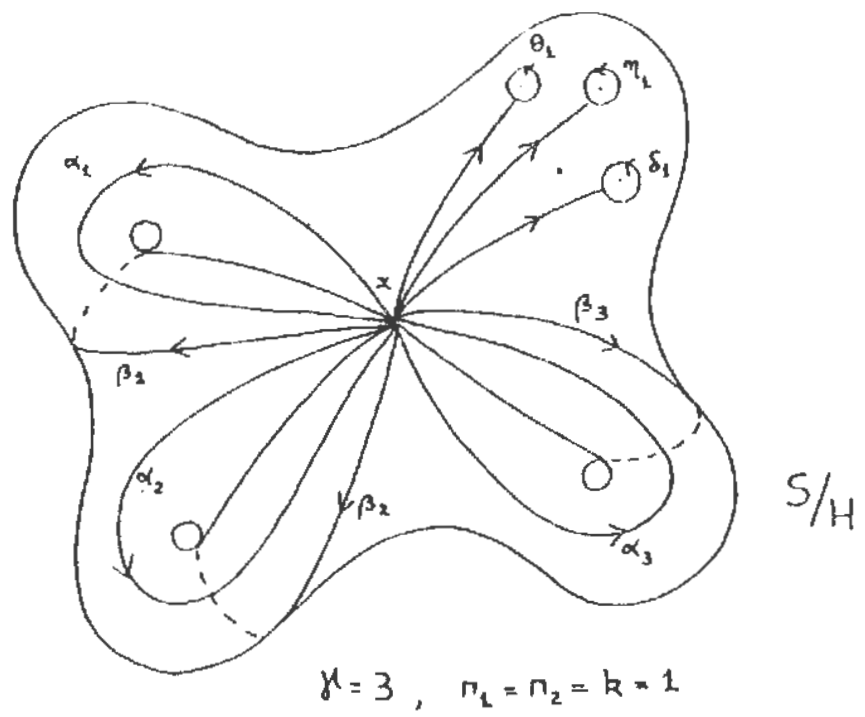

figure 1

Remark. Same arguments as in the proof of proposition 5.3 show that,

(1) if $\gamma=0$. these $r_{0} \neq 0$ a d $r_{1}+r_{2} \neq 0$.

(2) if there is no a subset of $\{1, \ldots, t\}$, say $\left\{i_{1}, \ldots, i_{k}\right\}$, satisfying the propertios that 
(2.1) the transformation $a^{y_{i}}$ generates the stabilizer of some point, and

$(2.2)$ the transformations $a^{y_{i_{1}}}, \ldots, a^{y_{i_{k}}}$ generate the cyclic subgroup of order $n$,

then $r_{0} \geq 2$ for $n$ even, and $r_{1}+r_{2} \geq 2$ for $n$ odd.

\section{A Special System of Simple Loops on $S / H$ in the Case $\gamma \geq 1$}

In this section we assume $\gamma$, the genus of the quotient Riemann surface $S / H$ to be greater or equal to one. Under this assumption we proceed to find a special set of simple loops, $\alpha_{i}, \beta_{i}(i=1, \ldots, \gamma)$ on $S / H$. This set of loops gives a canonical basis for the homotopy of $S / H$ and satisfies certain properties which describes the regular branched covering $\pi: S \rightarrow S / H$ (see proposition 6.3).

Let us fix $a$ and $b$ in $H$ of order $n$ and two, respectively. Denote by $S / L$ the quotient Riemann surface obtained by the action of $L$, the cyclic group generated by $a$, on the surface $S$. Let $\pi_{1}: S \rightarrow S / L$ and $\pi_{2}: S / L \rightarrow S / H$ be the natural holomorphic (branched) coverings induced by the actions of $L$ on $S$ and $H / L \cong \mathbf{Z} / 2 \mathbf{Z}$ on $S / L$, respectively. We have that $\pi=\pi_{2} \pi_{1}$. Let $b$ be the automorphism of $\mathrm{S} / L$ (of order two) generating $H / L$. Then $\pi_{1} a^{k} b=\bar{b} \pi_{1}$ all $k$.

Choose a point $x$ on $S / H$ which is not a branch value of $\pi$. Let $y_{1}$ and $y_{2}$ on $S / L$ be so that $\pi_{2}\left(y_{i}\right)=x$, for $i=1,2$. These points are necessarily no branch values of $\pi_{1}$. Let $z_{1}, \ldots, z_{n}, w_{1}, \ldots, w_{n}$ on $S$ be such that $\pi_{1}\left(z_{i}\right)=y_{1}$ and $\pi_{1}\left(w_{i}\right)=y_{2}$. We may assume that $a\left(z_{i}\right)=z_{i+1}, a\left(w_{i}\right)=w_{i+1}$, for $i=1, \ldots, n-1, a\left(z_{n}\right)=z_{1}, a\left(w_{n}\right)=w_{1}$ and $b\left(z_{1}\right)=w_{1}$.

Let $\alpha_{j}, \beta_{j}, j=1, \ldots, \gamma$, be a set of different oriented simple loops on $S / H$ through $x$ satisfying the following (see figure 2 ).

(A) The intersection of any two different loops is exactly $x$.

(B) The intersection number between $\alpha_{j}$ and $\beta_{j}$ is +1 .

(C) Any other pair of loops are homotopically disjoint, that is, they are homotopic: to disjoint simple loops.

(D) The set of loops above gives a canonical basis for the homotopy of $S / H$ (based at $x$ ).

(E) The kops are disjoint from the branch values of $\pi$. 


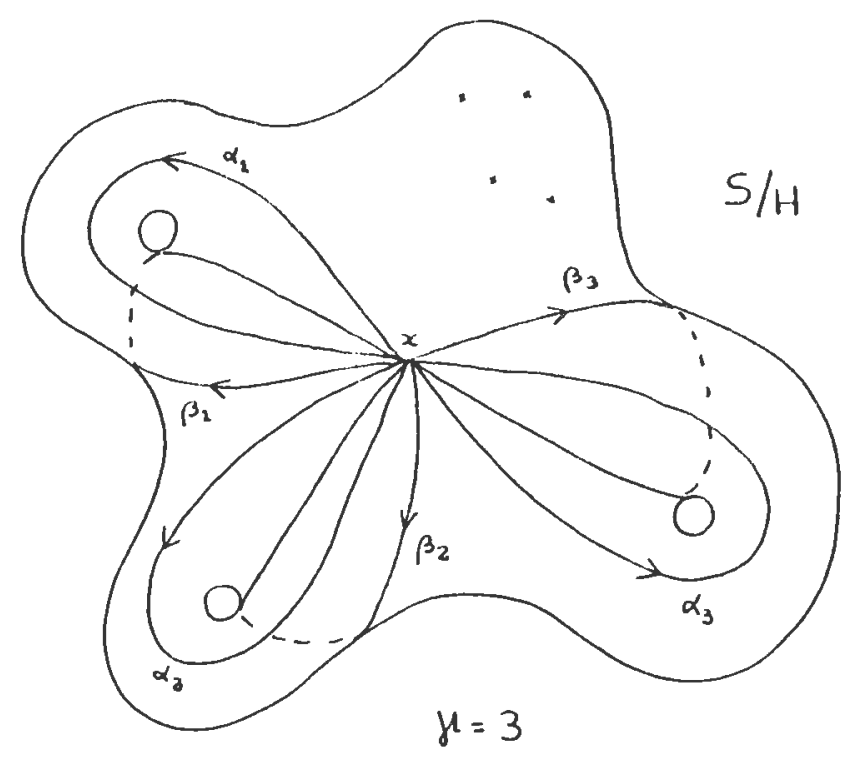

figure 2

\section{Lemma 6.1.}

(i) If some $a^{s} b$ has a fixed point, then we can choose the set of loops as above satisfying the property that all of them lift to loops on $S / L$.

(ii) If $a^{s} b$ has no fixed points for all $s$, then we can choose the above loops in such a way that $\alpha_{1}$ lifts to a path and all other loops lift to loops on $S / L$.

Proof Choose a set of oriented loops through $x$ satisfying (A) to (E). Each one of these loops either lifts to a loop or lifts to a path. Assume that some of these loops lifts to a path (this is the case if the automorphism $b$ 
acts free-fixed points), otherwise we are done. For each fixed $j$ we have the following possibilities.

(i) Both loops $\alpha_{j}$ and $\beta_{j}$ lift to loops.

(ii) One of the loop $\alpha_{j}$ or $\beta_{j}$ lifts to a loop and the other lifts to a path. By permuting them (if necessarily), we may assume that $\alpha_{j}$ lifts to a path.

(iii) Both loops $\alpha_{j}$ and $\beta_{j}$ lift to paths on $S / L$.

In case (iii) we can replace the loop $\beta_{j}$ for a simple loop homotopic: to $\alpha_{j}, 3_{j}$. After this change, we are in case (ii). From now on, we assume that for each $j$ we are either in situation (i) or (ii). We re-order the pairs of loops $\alpha_{j}$ and $\beta_{j}$ such that:

(1) $\alpha_{j}$ lifts to a path and $\beta_{j}$ lifts to a loop on $S / L$, all $j=1, \ldots, r$; and

(2) $\alpha_{j}$ and $\beta_{j}$ both lift to loops on $S / L$, all $j=r+1, \ldots, \gamma$.

For each $j, 2 \leq j \leq r$, we change the loops $\beta_{1}$ and $\alpha_{j}$ by simple loops homotopic: to $\beta_{1} \beta_{j}$ and $\alpha_{j} \alpha_{1}^{-1}$, respectively. After performing these changes, we have that $\alpha_{1}$ lifts to a path and all other loops lift to loops on $S / L$.

If there is no element $a^{s} b$ with a fixed point, then the automorphism $\bar{b}$ las no fixed points. The connectivity of the surface $S / L$ implies we cannot do better in this situation, and we obtain the required loops.

Now let us assume some $a^{s} b$ has fixed points. In this case, $\bar{b}$ has a fixed point. Denote it by $q$. Let $p$ be the projection of $q$ in $S / H$. Let $\eta$ be a small simple loop around $p$, disjoint from the above loops and from the branch locus of $\pi$. We may assume this loop separates $p$ from all other branch values of $\pi$. We orient $\eta$ in such a way that, when going in the positive orientation, the point $p$ is at the left side of $\eta$. Let $\delta$ be a simple path commecting $x$ to $\eta$. We assume this path to be disjoint, except at $x$, from the loops $\alpha_{j}$ and $\beta_{j}$, for all $j$. We orient this path so that $x$ is the initial point. Now replace the loop $\alpha_{1}$ by a simple loop homotopic to $\alpha_{1} \delta \eta \delta^{-1}$. The new set of loops is the required one.

Proposition 6.2. We can modify the above set of loops. without destroying the above properties, such that one of the following holds. 
(I) If $\gamma=1$, then either

(I.1) Both loops $\alpha_{1}$ and $\beta_{1}$ lift to loops on $S$; or

(I.2) The loop $\alpha_{1}$ lifts to a loop, and the loop $\beta_{1}$ lifts to a path with initial point $z_{1}$ and end point $z_{k}, k \in\{2, \ldots, n\}$ and $k-1$ dividing $n ; o r$

(I.3) The loop $\alpha_{1}$ lifts to a loop, and the loop $\beta_{1}$ lifts to a path with initial point $z_{1}$ and end point $w_{k}, k \in\{1, \ldots, n\}$; or

(I.4) The loop $\alpha_{1}$ lifts to a path with initial point $z_{1}$ and end point $z_{l}$, $l \in\{2, \ldots, n\}$, and the loop $\beta_{1}$ lifts to a path with initial point $z_{1}$ and end point $w_{k}, k \in\{1, \ldots, n\}$.

(II) If $\gamma \geq 2$, then either

(II.1) All the loops $\alpha_{i}$ and $\beta_{i}$ lift to loops on $S$; or

(II.2) All the loops $\alpha_{j}$ and $\beta_{k}$ lift to loops on $S$, for $j \geq 3$ and $k \geq 1$, the loop $\alpha_{1}$ lifts at $z_{1}$ with end point $z_{2}$, and the loop $\alpha_{2}$ lifts at $z_{1}$ with end point $w_{1}$; or

(II.3) The loop $\alpha_{1}$ lifts at $z_{1}$ with end point $z_{2}$, the loop $\alpha_{2}$ lifts at $z_{1}$ with end point $w_{1}$, the loop $\beta_{2}$ lifts at $z_{1}$ with end point at some $z_{l}$ and all the other loops lift to loops on $S$.

\section{Proof}

(I) The case $\gamma=1$. Let us call by $\alpha$ and $\beta$ the loops $\alpha_{1}$ and $\beta_{1}$ respectively. By lemma 6.1, either both lift to loops on $S / L$ or $\alpha$ lifts to a path and $\beta$ lifts to a loop on $S / L$. Change the loops $\alpha$ and $\beta$ by $\beta$ and $\alpha^{-1}$, respectively. Now $\alpha$ lifts to a loop and $\beta$ lifts either to a loop or to a path on $S / L$.

(1) Some automorphism $a^{s} b$ has fixed points. In this case both loops $\alpha$ and $\beta$ lift to loops on $S / L$. In this case, either both of them lift to loops on $S$ (in which case we are done) or

(i) The loop $\alpha$ lifts to a loop and the loop $\beta$ lifts at $z_{1}$ with end point $z_{l}$, $l \in\{2, \ldots, n\}$; or

(ii) the loop $\alpha$ lifts at $z_{1}$ with end point $z_{l}$ and $\beta$ lifts at $z_{1}$ with end point $z_{k}, l, k \in\{2, \ldots, n\}$. 
In case (ii), we consider the group generated by $a^{l-1}$ and $a^{k-1}$. This gromp is a cyclic: group generated by $a^{h}$ with $h$ dividing $n$. In particular, there exist $i$ and $j$ integers, such that $a^{i(l-1)+j(k-1)}=a^{h}$. Let $r$ be the greater common divisor of $l-1$ and $k-1$. In this case we have:

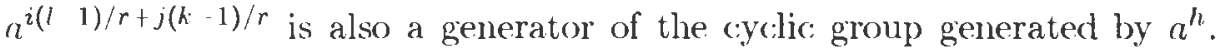
Since $(l-1) / r$ and $(k-1) / r$ are relatively prime, there exists a simple loop $\eta$ through $x$; generated by $\alpha$ and $\beta$ which lifts at $z_{1}$ with end point $a^{v-1}\left(z_{1}\right)$, where $v=i(l-1) / r+j(k-1) / r$. Let $\delta$ be any simple loop on $S / H$ through $x$, generated by $\alpha$ and $\beta$, such that $\eta$ and $\delta$ satisfy the properties (A) to (E) as above. There exists an integer $m$ such that $\eta^{m}$ lifts at $z_{1}$ with the same end point as $\delta$. Change the loops $\alpha$ and $\beta$ by $\eta^{-m} \delta$ and $\eta$. Now we are in case (i).

Similar arguments, as done above, can be use to show that we may assume that $l-1$ divides $n$ in case (i).

$(2)$ The automorphisms of the form $a^{s} b$ have no fixed points. In this case, lemma 6.1 asserts that the loop $\alpha$ lifts to a loop and the loop $\beta$ lifts to a path on $S / L$. The loop $\beta$ must lift to a path on $S$ with initial point $z_{1}$ and end point $w_{k}$, for some $k \in\{1, \ldots, n\}$. The loop $\alpha$ either lifts to a loop on $S$ or it lifts to a path with initial point $z_{1}$ and end point $z_{l}$, for some $l \in\{2, \ldots, n\}$. This finish the case $\gamma=1$.

(II) The case $\gamma \geq 2$. From now on, we assume our loops to satisfy one of the possibilities given in lemma 6.1. Assume also that some of these hoos lifts to a path on $S$, otherwise we are in condition (II.1) of our proposition.

Let us denote by $\alpha_{j, 1}, \alpha_{j, 2}, \beta_{j, 1}$ and $\beta_{j, 2}$ the liftings of $\alpha_{j}$ and $\beta_{j}$ to $s / L$ respectively, all $j=2, \ldots, \gamma$. We assume $\alpha_{j, 1}$ and $\beta_{j, 1}$ to be the liftings at $y_{1}$ and $\alpha_{j, 2}$ and $\beta_{j, 2}$ to be the liftings at $y_{2}$. Since the loops $\alpha_{j}$ and $\beta_{j}$ are simple, the intersection number of them is +1 , the intersection number of two other loops is zero and the covering $\pi_{2}$ is two to one, we have the following:

(1) The loops $\alpha_{j, 1}$ and $\alpha_{j, 2}$ are disjoint.

()ㅡ The forops $3_{j, 1}$ and $\beta_{j, 2}$ are disjoint.

(3) The jntersection number between $\alpha_{j, i}$ and $\beta_{j, i}$ is +1 , and all other intersection numbers are zero.

(4) $\bar{b}\left(\alpha_{j .1}\right)=\Omega_{j .2}$ and $\bar{b}\left(\beta_{j, 1}\right)=\beta_{j .2}$.

We (an choose the loops such that $\alpha_{2}$ lifts to $S$ to a path at $z_{1}$ with end point $z_{2}$ and all other loops lift to loops on $S$. In fact, from the above 
observations, we only need to find these loops such that $\alpha_{2,1}$ lifts to a path on $S$ at $z_{1}$ with end point at $z_{2}$ and the loops $\alpha_{j, 1}$ and $\beta_{k, 1}$ all lift to loops on $S$, for $j=3, \ldots, \gamma$ and $k=2, \ldots, \gamma$. We are in the cyclic case, done in [6]. Let us see now the loops $\alpha_{1}$ and $\beta_{1}$. We have two rases.

(1) Some automorphism $a^{s} b$ has fixed points. In this case the loops $\alpha_{1}$ and $\beta_{1}$ both lift to loops on $S / L$. We can proceed as above to change them so that both lift to loops on $S$. In this case we permute the pair $\alpha_{1}$ and $\beta_{1}$ with the pair $\alpha_{2}$ and $\beta_{2}$. Since some element of order two in $H$ has a fixed point, either $b$ has a fixed point or $a b$ has a fixed point. Denote by $q$ such a fixed point and denote by $p$ its projection to $S / H$. Let $\eta$ be a small simple loop around $p$, disjoint from the above loops and from the branch locus of $\pi$. We may assume this loop separates $p$ from all other branch values of $\pi$. We orient $\eta$ in such a way that, when going in the positive orientation, the point $p$ is at the left side of $\eta$. Let $\delta$ be a simple path connecting $x$ to $\eta$. We assume this path to be disjoint, except at $x$, from the loops $\alpha_{j}$ and $\beta_{j}$, for all $j$. We orient this path so that $x$ is the initial point. Now replace the loop $\alpha_{2}$ by a simple loop homotopic to $\alpha_{2} \delta \eta \delta^{-1}$.

At this point, if the point $q$ is a fixed point of $b$, we are done. Assume the point $q$ is a fixed point of $a b$. We replace the loops $\beta_{1}$ and $\alpha_{2}$ by simple loops homotopic: to $\beta_{1} \beta_{2}$ and $\alpha_{2} \alpha_{1}^{-1}$, respectively. We get in this way condition (II.2) of our proposition.

(2) The automorphism $a^{s} b$ has no fixed points for every $s$. In this case, the loop $\alpha_{1}$ lifts to a path connecting $y_{1}$ to $y_{2}$ and the loop $\beta_{1}$ lifts to a loop on $S / L$. Denote by $\alpha_{1,1}$ the lifting of $\alpha_{1}$ at $y_{1}$, by $\alpha_{1,2}$ the lifting of the same loop at $y_{2}$, by $\beta_{1, i}$ the lifting of $\beta_{1}$ at $y_{i}$, for $i=1,2$.

We have two possibilities for the liftings of $\beta_{1}$ on $S$. Either it lifts to a loop on $S$ or it lifts to a path at $z_{1}$ with end point some $z_{l}$, for some $1 \leq l \leq n-1$.

Since the loop $\alpha_{1}$ does not lift to a loop on $S / L$, it must lift on $S$ to a path at $z_{1}$ with end point $w_{k}$, for some $k$.

If $\beta_{1}$ lifts to a loop on $S$, we replace the loops $\alpha_{1}, \alpha_{2}$ and $\beta_{2}$ by simple loops homotopic: to $\alpha_{2}^{n-k+1} \alpha_{1}^{-1} \beta_{2}^{-1}, \alpha_{2} \beta_{2} \beta_{1} \beta_{2}^{-1}$ and $\beta_{2} \alpha_{2}^{-n+k-1}$, respec:- 
tively (see figure 3).
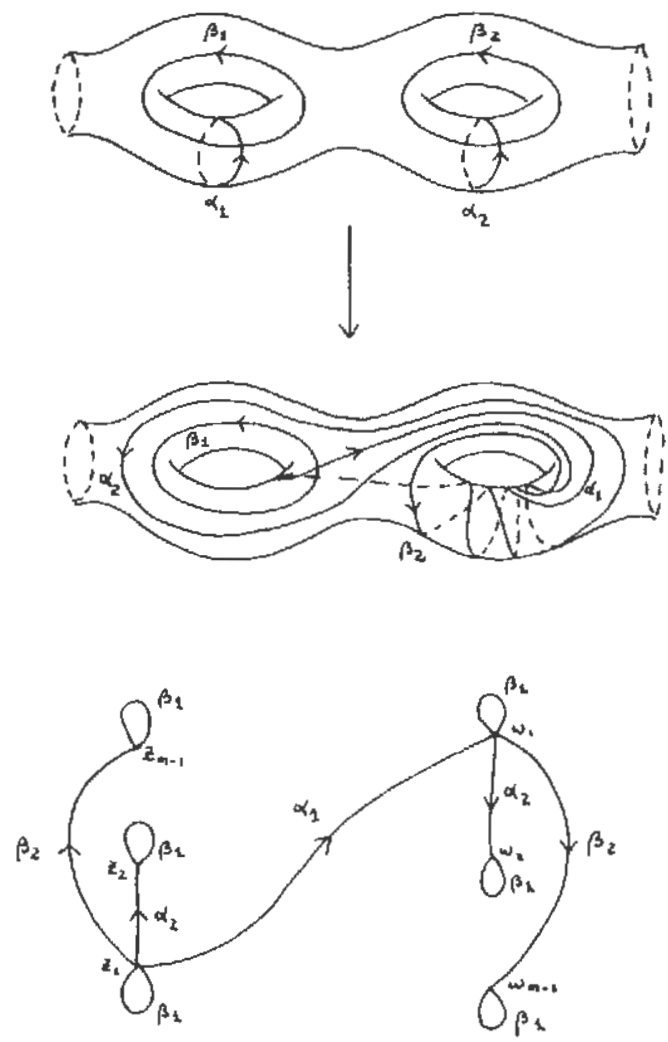

figure 3 
Now replace the loop $\beta_{2}$ by a simple loop homotopic to $\beta_{2} \alpha_{2}^{n-k+1}$. At this point we have that the loop $\alpha_{1}$ lifts to a path connecting $z_{1}$ to $w_{1}$, the loop $\alpha_{2}$ lifts to a path at $z_{1}$ with end point $z_{2}$, and all other loops lift to loops on $S$. Permute the pair of loops $\alpha_{1}$ and $\beta_{1}$ with the pair $\alpha_{2}$ and $\beta_{2}$. We obtain condition (II.2) of our proposition.

If the loop $\beta_{1}$ lifts to a path at $z_{1}$ with end point $z_{l}$, for some $1 \leq$ $l \leq n-1$, then we replace the loops $\alpha_{1}, \beta_{1}, \alpha_{2}$ and $\beta_{2}$ by simple loops homotopic to $\alpha_{1} \alpha_{2}{ }^{1}, \beta_{1}, \alpha_{2}$ and $\beta_{1} \alpha_{2}^{-l+1} \beta_{2}$ respectively. We have the same situation as before but now $\alpha_{1}$ lifts to a path at $z_{1}$ with end point $w_{k+1}$. If we do the same procedure again $n-k$ times more, we obtain that $\alpha_{1}$ lifts at $z_{1}$ and ends at $w_{1}$. Let us permute the pair $\alpha_{1}, \beta_{1}$ with the pair $\alpha_{2}, \beta_{2}$. Now we are in condition (II.3).

In the arguments above we have fixed two elements $a, b$ in $H$ of orders $n$ and 2 , respectively. For each such a pair, proposition 6.2 gives us a set of special loops satisfying certain possibilities. The next proposition asserts that we can modify the pair $a$ and $b$ (with the same properties) so that these special loops satisfy only few of them.

Proposition 6.3. Let $a$ and $b$ be two elements in $H$ of order $n$ and 2, respectively. Let $\alpha_{1}, \ldots, \alpha_{\gamma}, \beta_{1}, \ldots, \beta_{\gamma}$, be the loops found in proposition 6.2. Then we can change the generators $a$ and $b$ for new ones, say $\tilde{a}$ and $\tilde{b}$ of order $n$ and 2, respectively, so that we have one of the following situations:

(I) If $\gamma=1$, then the loop $\alpha_{1}$ lifts to a path with initial point $z_{1}$ and end point $z_{2}=\tilde{a}\left(z_{1}\right)$, the loop $\beta_{1}$ lifts to a path starting at $z_{1}$ and ending at $w_{1}=\tilde{b}\left(z_{1}\right)$.

(II) If $\gamma \geq 2$, then $\alpha_{1}$ lifts to a path starting at $z_{1}$ and ending at $z_{2}=\tilde{a}\left(z_{1}\right)$. $\alpha_{2}$ lifts to a path starting at $z_{1}$ and ending at $w_{1}=\tilde{b}\left(z_{1}\right)$. The other. loops lift to loops.

\section{Proof}

The case $\gamma=1$. (1) Assume we are in case (I.1) of proposition 6.2. In this case, we must have fixed points for $b$ or $a b$. In either case, we can change the generators $a$ and $b$ by the generators $a=a$ and $a b$. In particular, we may assume that $b$ has fixed points. We can modify the loop $\beta_{1}$ such that its lifting at $z_{1}$ ends at some $w_{k}$. In fact, choose a small simple loop $\delta$ around the projection of some fixed point of $b$. Let $\eta$ be a simple path 
comecting $\beta_{1}$ to $\delta$. We orient eta so that it ends at $\delta$ and we orient $\delta$ so that, going in the positive way, the branch point it encloses is at the left side. We may assume the above loops to be disjoint from the loop $\alpha_{1}$. Now replace $\beta_{1}$ by a simple loop homotopic to $\beta_{1} \eta \delta \eta^{-1}$. We are in case (I.3) to be andyzed later.

(2) Assume we are in case (I.2) of proposition 6.2. We must have fixed points of $b$ or $a b$. Now we can proceed as in (1).

(3) If we are in case (I.4) of proposition 6.2. Assume first that neither $b$ nor $a b$ to have fixed points. In this case, the transformations $a^{l-1}$ and $a^{k} \quad{ }^{1} b$ generate $H$. We change the generators $a$ and $b$ by $a^{l-1}$ and $a^{k-1} b$, respectively. The loop $\alpha_{1}$ lifts at $z_{1}$ with end point $z_{2}$ and $\beta_{1}$ lifts at $z_{1}$ with end point $w_{1}$. At this point, we are in situation (I.4) with $l=2$ and $k=1$. Let us assume now that $b$ or $a b$ has fixed points. By replacing $b$ by $a b$ if necessary, we may assume $b$ has fixed points. Using the same idea as in (1), we change the loop $\beta_{1}$ so that it lifts at $z_{1}$ with end point $z_{l}$, for some $l$. We change again the loops $\alpha_{1}$ and $\beta_{1}$ so that $\alpha_{1}$ lifts to a loop and $\beta_{1}$ lifts at $z_{1}$ with end point $z_{r}$, for some. $r$. Change the loop $\beta_{1}$ as in (1), so that it lifts at $z_{1}$ with end point some $w_{s}$. Now we are in case (I.3) of proposition 6.2 , to be analyzed below.

(4) In case (I.3) of proposition 6.2, we change the pair of generators $a$ and $b$ by the generators $a$ and $a^{k-1} b$ respectively. Let us call them again $a$ and $b$, respectively. In this case the loop $\alpha_{1}$ lifts to a loop and the loop $\beta_{1}$ lifts at $z_{1}$ with end point $=b\left(z_{1}\right)=w_{1}$ for the new pair $a$ and $b$ of generators of $H$. The comnectivity of the surface $S$ asserts that is there a fixed point of either $a$ or $a^{t} b$ for some $t \in\{1, \ldots, n-1\}$. Denote by $q \in S / H$ the projection of one such fixed point. Consider a small oriented simple loop $\eta$ around $q$ so that it separates $q$ from the other possible branched values. Take an oriented simple path $\delta$ starting from the intersection point $x$ between $\alpha_{1}$ and $\beta_{1}$ and ending at a point in $\eta$. We may assume such a path to be disjoint from the branching set and from $\left(\alpha_{1} \cup \beta_{1}\right)-\{x\}$. Now replace the loop $\alpha_{1}$ by $\delta \eta \delta^{-1} \alpha_{1}$. Now the new loops satisfy the desired properties.

The case $\gamma \geq 2$. In this case, we have three possibilities marked in proposition 6.2 as (II.1), (II.2) and (II.3). In case (II.1), we may proceed in sinilar way as in the case $\gamma=1$ to obtain the case (II.2). In case (II.3), we proceed as follows. Consider a small oriented simple loop $\eta$ on $S / H$ bounding a topological disc containing all branch values. This loop is free homotopic to the loop $\prod_{i=1}^{\gamma}\left(\alpha_{i} \beta_{i} \alpha_{i}^{-1} \beta_{i}^{-1}\right)$. In particular, the loop $\eta$ lifts at $z_{1}$ and ends at $a^{l-1} b a^{1-l}\left(z_{1}\right)=a^{2 l-2}\left(z_{1}\right)$. Consider a simple oriented path 
$\delta$ starting at $x$ and ending at a point in $\eta$. This path may be assumed to be disjoint from all other loops (except for $x$ ). Then either $\left.\beta_{2} \delta \eta\right)^{-1} \delta^{-1}$ or $\beta_{2} \delta \eta^{-1} \delta^{-1} \alpha_{2}^{-1}$ is homotopic to a simple loop. If $\beta_{2} \delta \eta^{-1} \delta^{-1}$ is the one homotopis: to a simple loop, then we replace $\beta_{2}$ by this new loop. In the second case, the loop $\beta_{2} \delta \eta^{-1} \delta^{-1} \alpha_{2}{ }^{1}$ lifts at $z_{1}$ and ends at $w_{1}=b\left(z_{1}\right)$. We replace the loop $\beta_{2}$ by the loops $\beta_{2} \alpha_{2}^{-1} \delta \eta^{-1} \delta^{-1} \alpha_{2}^{-1}$ (see figure 4).
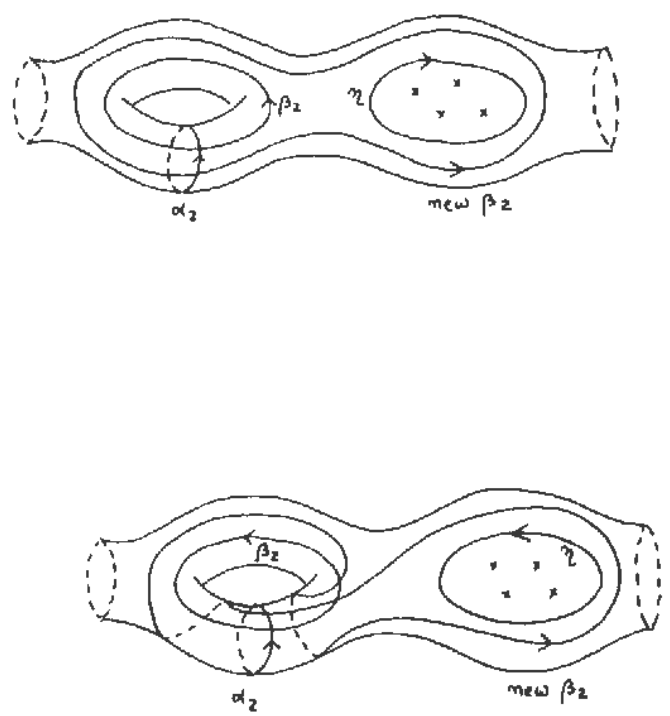

figure 4 


\section{Proof of Theorem 4.1.}

In this section, we proceed to prove theorem 4.1. concerning free fixed point actions of diledral groups. Let us assume we have:

(1) a closed Riemann surface $S$ of gemus $g \geq 2$,

(2) a group $H$ of conformal automorphisms of $S$,

(3) $H$ isomorphic: to a dihedral group of $\operatorname{order} 2 n$, and

(4) the action of $H$ on $S$ is free fixed points.

Let us denote by $R$ the quotient Riemann surface $S / H$ and by $\pi: S \rightarrow R$ the natural holomorphic unbranched covering induced by the action of $H$ on $S$.

The genus $\gamma$ of $R$ satisfies, by Riemann-Hurwitz formula,

$$
\gamma=\frac{g-1}{2 n}+1 \geq 2
$$

Part (II) of proposition 6.3. asserts that we may find $\gamma$ disjoint, oriented, homologically independent simple loops, say $\beta_{1}, \ldots, \beta_{\gamma}$, with the property that the lifting of each one of them, at any point, is a loop.

We claim that the family $\mathcal{F}$ of loops on $S$, obtained by lifting the $\gamma$ loops $\beta_{1}, \ldots, \beta_{\gamma}$, disconnects $S$ into genus zero surfaces. Before to prove it, let us finish the proof of theorem 4.1.

Since the family $\mathcal{F}$ cuts $S$ into genus zero surfaces, we may find a subfamily $\mathcal{G} \subset \mathcal{F}$ formed by $g$ disjoint homologically independent simple loops. The families $\mathcal{G}$ and $\mathcal{F}$ have the same normalizer $\mathcal{N}$ in the fundamental group of $S$. Since the family $\mathcal{F}$ is invariant under the action of $H$, then $\mathcal{N}$ is also invariant under $H$. It follows that the Schottky uniformization determined by the family $\mathcal{G}$ (Retrosection theorem) is the desired one.

Now we proceed to prove the above claim. Let $X$ be a comected component of $S-\mathcal{F}$. Set $R=S / H-\left\{\beta_{1}, \ldots, \beta_{\gamma}\right\}$. We have the restriction $P: X \rightarrow R$, of the covering $\pi: S \rightarrow S / H$. This is again a covering, induced by a subgroup of $H$, the subgroup generated by those elements keeping $X$ invariant. The order of this subgroup, say $d$, is the degree of $P$.

By Maskit [15], we may assume that $X$ is the complement of $k$ disjoint circular discs on a closed Riemann surface $\tilde{X}$ of genus $\tilde{g}$ and, $R$ is the complement of $\gamma$ disjoint circular discs on the Riemann sphere $\mathbf{C}$. 
The property that each loop $\beta_{i}$ lifts to a loop, permits to extend the covering $P: X \rightarrow R$ to an unbranched covering $\tilde{P}: \tilde{X} \rightarrow \hat{\mathbf{C}}$, of degree $d$.

Riemann-Hurwitz formula applied to the above covering asserts that $\tilde{g}=1-d$. Since $\tilde{g} \geq 0$ and $d \geq 1$, we must have $d=1$ and $\tilde{g}=0$. In particular, the surfaces $X$ and $R$ are conformally equivalent by $P: X \rightarrow R$.

We can describe explicitly the above construction as follows. The family $\left\{\beta_{1}, \ldots, \beta_{\gamma}\right\}$ and Retrosection theorem assert that there exist:

(1) a Schottky covering $(\Omega, K, P: \Omega \rightarrow R)$ of $R$,

(2) a set of free generators $B_{1}, \ldots, B_{\gamma}$ for $K$,

(3) a set of $2 \gamma$ disjoint simple loops, $\eta_{1}^{+}, \eta_{1}^{-}, \ldots, \eta_{\gamma}^{+}, \eta_{\gamma}^{-}$, bounding a common domain $\mathcal{D}$ of connectivity $2 \gamma$, such that:

(3.1) $B_{i}\left(\eta_{i}^{+}\right)=\eta_{i}^{-}$,

(3.2) $B_{i}(\mathcal{D}) \cap \mathcal{D}=\emptyset$.

(3.3) $P\left(\eta_{i}^{+}\right)=\beta_{i}$.

If $G$ is the smallest normal subgroup of $K$ containing the elements

$$
B_{1}^{n}, B_{2}^{2},\left(B_{2} B_{1}\right)^{2}, B_{3}, \ldots, B_{\gamma}
$$

then $G$ turns out to be a Schottky group of genus $g$ uniformizing the surface $S$ as desired.

\section{A Counterexample}

In this section, we construct a closed Riemann surface $S$ of genus 4 and a group $H$ of conformal automorphisms of $S$, isomorphic to a dihedral group of order six, which cannot be lifted to any Schottky covering of $S$. It follows that, in the presence of fixed points, the condition (A) in [4] is not sufficient for the class of dihedral groups.

9.1. Construction of a dihedral action. Let us consider the dihedral group of order six

$$
D_{3}=\left\langle a, b ; a^{3}=b^{2}=(a b)^{2}=1\right\rangle,
$$

and the Fuchsian group

$$
\Gamma=\left\langle A_{1}, A_{2}, A_{3}, B_{1}, B_{2} ; A_{1}^{3}=A_{2}^{3}=A_{3}^{3}=B_{1}^{2}=B_{2}^{2}=A_{1} A_{2} A_{3} B_{1} B_{2}=I\right\rangle,
$$


uniformizing a Riemann surface $R$ of gemus zero with signature $(0,5 ; 2,2,3,3.3)$, acting on the liyperbolic plane $\mathbf{H H}$. Denote by $\pi_{\Gamma}: \mathbf{H H} \rightarrow R$ the holomorphic: (minversal) branched covering of $R$ induced by $\Gamma$. Its branched values are $p_{1}, p_{2}, q_{1}, q_{2}$ and $q_{3}$ with branch values $2,2,3,3$, and 3 , respectively.

We lave a surjective homomorphism

$$
\phi: \Gamma \rightarrow D_{3}
$$

defined by: $\phi\left(A_{i}\right)=a$ and $\phi\left(B_{j}\right)=b$, for $i=1,2,3$ and $j=1,2$.

Let us denote by $F$ the kernel of this homomorphism. Then we have that $F$ is torsion free and it corresponds to the smallest normal subgroup of $\Gamma$ containing the words:

$$
B_{2} B_{1}, A_{2} A_{1}, A_{3} A_{1},\left(A_{1} B_{1}\right)^{2} .
$$

Following unifpack calculator [17], we have that:

Generators of $\mathbf{F} \quad C_{1}=A_{3} A_{1}^{-1}, C_{2}=A_{3}^{-1} A_{1}^{-1} A_{3}^{-1}, C_{3}=A_{3} A_{2}^{-1}, C_{4}=$ $A_{3}^{-1} A_{2}^{-1} A_{3}^{-1}, C_{5}^{-}=A_{3} B_{1} A_{1} B_{1}, C_{6}=A_{3}^{-1} B_{1} A_{1} B_{1} A_{3}^{-1}, C_{7}=A_{3} B_{1} A_{2} B_{1}$, $C_{8}=A_{3}^{-1} B_{1} A_{2} B_{1} A_{3}^{-1}$;

Relation $C_{3} C_{6} C_{5} C_{7}^{-1} C_{1} C_{3}^{-1} C_{4}^{-1} C_{6}^{-1} C_{8} C_{7} C_{1}^{-1} C_{2}^{-1} C_{4} C_{5}^{1} C_{8}^{--1} C_{2}=I$.

If we set $S=\mathbf{H H} / F$, then we have a natural holomorphic (universal) covering $\pi_{F}: \mathbf{H H} \rightarrow S$, such that

$$
\phi(\gamma) \pi_{F}=\pi_{F} \gamma,
$$

for all $\gamma \in \Gamma$. It follows that $H=\Gamma / F \cong D_{3}$ acts on $S$ as group of conformal antomorphism. We have then a natural holomorphic (branched) covering $\pi: S \rightarrow R$, induced by the action of $H$, branched over $p_{1}, p_{2}, q_{1}, q_{2}$ and $q_{3}$ with branch values $2,2,3,3$, and 3 , respectively. In particular, $\pi \pi_{F}=\pi_{\Gamma}$. The Riemann-Hurwitz formula asserts that the genus of $S$ is $g=4$.

9.2. The non lifting property。 Assume there is a Schottky covering $(\Omega, G . P: \Omega \rightarrow S)$ for which the group $H$ lifts. Let us denote by $J$ the geometrically finite Kleinian group obtained by lifting $H$ via the above covering. We have a regular branched covering $Q: \Omega \rightarrow R$ such that $Q=\pi P$.

By construction, we have an oriented simple loop $\alpha$ on $R$ (disjoint from the branch vahues), bounding a topological disc: $\Delta_{1}$ containing on its interior the branch values $p_{1}, p_{2}$ and no other, and lifting to a simple loop on $S$. The loop a corresponds in $\Gamma$ to the transformation $B_{1} B_{2}$. Set $\Delta_{2}$ the other topological disc bounded by $\alpha$ on $R$.

IVe have that 
(A) each comnected component of $\pi^{-1}\left(\Delta_{1}\right)$ is a cylinder, invariant under the action of an element $b$ of order two in $H$, and the two boundary loops (each one a lifting of $\alpha$ ) are permuted by it.

(B) $\pi^{-1}\left(\Delta_{2}\right)$ is either (using Riemann-Hurwitz formula)

(B.1) connected and topologically a surface of genus one with six boundary loops (the liftings of $\alpha$ ), invariant under the action of $H$ and the cyclic group acting with six fixed points; or

(B.2) two connected components, each one a genus one surface with three boundaries components and invariant under the cyclic: group of order three (of $H$ ) acting with exactly three fixed points.

If the loop $\alpha$ lifts to a loop on $\Omega$. Let $\mathcal{A}$ be a connected component of $Q^{-1}\left(\Delta_{2}\right)$ and set $\mathcal{B}=P(\mathcal{A})$. We have that $P(\mathcal{A})$ is a connected component of $\pi^{-1}\left(\Delta_{2}\right)$.

Set $J_{\mathcal{A}}=\{j \in J ; j(\mathcal{A})=\mathcal{A}\}$, that is, the stabilizer of $\mathcal{A}$ in $J$. We have that $J_{\mathcal{A}}$ is necessarily finitely generated $\left(\mathcal{A} / J_{\mathcal{A}}\right.$ is the finite surface $\left.\Delta_{2}\right)$. A finitely generated subgroup of a geometrically finite Kleinian group (of second kind) is necessarily geometrically finite by a result of Thurston [16].

Set $G_{\mathcal{A}}=G \cap J_{\mathcal{A}}$. This is finitely generated $\left(\mathcal{A} / G_{\mathcal{A}}\right.$ is the finite surface $\pi^{-1}\left(\Delta_{2}\right)$ ) and geometrically finite by Thurston' result, purely loxodromic: and free group (a subgroup of $G$, a purely loxodromic: and free group). It follows by Maskit's results [13] that $G_{\mathcal{A}}$ is a Schottky group. Moreover, $G_{\mathcal{A}}$ is normal subgroup of $J_{\mathcal{A}}$.

If $\Omega_{\mathcal{A}}$ is the region of discontimuity of $J_{\mathcal{A}}$, also for $G_{\mathcal{A}}$, we have natural holomorphic (branched) coverings $Q_{\mathcal{A}}: \Omega_{\mathcal{A}} \rightarrow \Omega_{\mathcal{A}} / J_{\mathcal{A}}=R_{\mathcal{A}}, P_{\mathcal{A}}: \Omega_{\mathcal{A}} \rightarrow$ $\Omega_{\mathcal{A}} / G_{\mathcal{A}}=S_{\mathcal{A}}$ and $\pi_{\mathcal{A}}: S_{\mathcal{A}} \rightarrow R_{\mathcal{A}}$, such that $Q_{\mathcal{A}}=\pi_{\mathcal{A}} P_{\mathcal{A}}$. The surfaces $R_{\mathcal{A}}$ and $S_{\mathcal{A}}$ are a gemus zero surface (the surface $\Delta_{2}$ after gluing a topological disc to it about $\alpha$ ) and a genus one surface (the surface $B$ after gluing topological discs to it about its boundaries).

At this point, we have a Schottky covering of the genus one surface $S_{\mathcal{A}}$ for which eitler:

(i) we can lift a cyclic group of order three, with exactly three fixed points, acting as group of conformal automorphisms of $S_{\mathcal{A}}$; or

(ii) we can lift a dihedral group of order six, where the cyclic group acts with fixed points.

In case (i), we are in contradiction to the results in [4] and [6]. In case (ii), we are contradiction to theorem 4.1. 
If the loop $\alpha$ lifts to a path on $\Omega$. Since $\alpha$ lifts to a loop on $S$, we liave that the stabilizer of any comnected component of $Q^{-1}(\alpha)$ is necessarily a cyclic: subgroup of $G$. Moreover, since $\pi^{-1}\left(\Delta_{1}\right)$ is a cylinder where both boundaries are liftings of $\alpha$, we must have that another connected components of $Q^{-1}(\alpha)$ so that each two have the same stabilizer, say the (yclic: group generated by the primitive loxodromic transformation $g$. In particular, each pair bounds a topological disc: on the Riemann sphere (one component start at one fixed point $a(g)$ of $g$ arriving to the other fixed point $r(g)$, and the other component start at $r(g)$ and arrives to $a(g)$. This disc is one of the connected components of $Q^{-1}\left(\Delta_{1}\right)$.

We nust have a transformation $j \in J$ that sends one of these components to the other. In particular, we must have $(j g)^{2}=I$, where $I$ is the identity of $J$. The transformation permutes both fixed points of $g$, so a transformation of order two, $j^{2}=I$. The stabilizer of the topological disc: bounded by these two components is the infinite dihedral group generated by $g$ and $j$.

Since each connected component of $Q^{-1}\left(\Delta_{1}\right)$ is simply connected, we have that $X=Q^{-1}\left(\Delta_{2}\right)$ is connected. In particular, $P(X)=\pi^{-1}\left(\Delta_{2}\right)$ is connected.

It follows that $\pi^{-1}\left(\Delta_{2}\right)$ is a genus one surface with six boundary components, invariant under the action of $H$, and the cyclic group of order three acting with exactly six fixed points. Moreover, the boundaries are permuted and no one is invariant under such a cyclic group. The arguments done in section 3 assert that this situation is impossible (there is no automorphism of order three on a surface of genus one with exactly six fixed points.

\section{A list of Geometrically finite Kleinian groups}

Let us consider $n \geq 3$ and the dihedral group

$$
D_{n}=\left\langle a, b ; a^{n}=b^{2}=(a b)^{2}=1\right\rangle .
$$

In this section, we construct geometrically finite Kleinian groups (of second kind) $J$ containing a Schottky group ( $r$ as a normal subgroup such that $J / G$ is isomorphic to $D_{n}$.

If we denote by

(1) $\Omega$ the region of discontinuity of such a group $J$,

(2) $R$ tlie quotient Riemann surface $\Omega / J$, 
(3) $S$ the quotient Riemann surface $\Omega / G$,

(4) $Q: \Omega \rightarrow R$ the natural (branched) holomorphic covering induced by $J$, and

(5) $P: \Omega \rightarrow S$ the natural holomorphic covering induced by $G$,

then $H=J / G$ is a group of holonorphic automorphisms isomorphic: to $D_{n}$, which can be lifted to the Schottky covering $(\Omega, G, P: \Omega \rightarrow S)$. The lifting of $H$ is then $J$. We have that the natural (branched) holomorphic: covering $\pi: S \rightarrow S$, induced by the group $H$, satisfies $Q=\pi P$.

10.1. Some necessary conditions for the constructions. First of all, let us assume that we may construct such an example. We proceed to find some conditions, necessary for the constructions if $n$ is a prime integer.

In this case, $N(b)=2 r$ and $N(a)=2 k$. The signature of the quotient Riemann surface $R$ has the form (from section 5 )

$$
(\gamma, 2 r+k ; 2, \ldots, 2, n, \ldots, n)
$$

where there are $2 r$ numbers 2 and $k$ numbers $n$.

As a consequence of propositions 6.2 and 6.3 , we may assume that there are a set of simple loops $\alpha_{1}, \ldots, \alpha_{\gamma}, \beta_{1}, \ldots, \beta_{\gamma}$ (based at some point $x \in R$ ), with the properties described in proposition 6.3.

Let $z_{i}$ and $w_{i}, i=1, \ldots, n$, be the liftings on $S$ of $x$ under the holomorphic (branched) covering $\pi: S \rightarrow S / H$. As before, we may assume that $a\left(z_{i}\right)=$ $z_{i+1}, a\left(w_{i}\right)=w_{i+1}$, and $b\left(z_{1}\right)=w_{1}$. In this way, we have the following possible signatures in each case.

The case $\gamma=0$ 。 The only possible signatures are:

(1) $(0,2 r ; 2, \ldots, 2), r \geq 2$, and

(2) $(0,2 r+k ; 2, \ldots, 2, n, \ldots, n), k \geq 1$ and $r \geq 1$.

The case $\gamma=1$. In this case, the loop $\alpha_{1}$ lifts to a path with initial point $z_{1}$ and end point $z_{2}$, and the loop $\beta_{1}$ lifts to a path with initial point $z_{1}$ and end point $w_{1}$. The only possible signatures are:

(1) $(1,2 r ; 2, \ldots, 2), r \geq 1$.

(2) $(1,2 r+k ; 2, \ldots, 2, n, \ldots, n), k \geq 1$ and $r \geq 0$. 
The case $\gamma \geq 2$. In this case, the loops $\alpha_{j}$ and $\beta_{i}$ lift to loops, for $j \geq 3$ and all $i$, the loop $\alpha_{1}$ lifts to a path with initial point $z_{1}$ and end point $z_{2}$, and the loop $\alpha_{2}$ lifts to a path with initial point $z_{1}$ and end point $w_{1}$. The possible signatures in this case are:

(1) $(\gamma, 0 ;-)$.

(2) $(\gamma, 2 r ; 2, \ldots \cdot 2), r \geq 1$.

(3) $(\gamma, 2 r+k ; 2, \ldots, 2, n, \ldots, n), k \geq 1$ and $r \geq 0$.

10.2. Sonne Möbius transformations. To construct our examples, we need the following Möbius transformations. Let $A, B$ and $($ ' be the following:

$$
A=\left(\begin{array}{cc}
\epsilon^{\pi i / n} & 0 \\
0 & \epsilon^{-\pi i / n}
\end{array}\right), \quad B=\left(\begin{array}{cc}
0 & i \\
i & 0
\end{array}\right), \quad C=\left(\begin{array}{cc}
\lambda & 0 \\
0 & \lambda^{-1}
\end{array}\right),
$$

where $\lambda$ is a real number greater than one.

Denote by $A^{p / q}$ the Möbius transformation

$$
A^{p / q}=\left(\begin{array}{cc}
e^{\pi i p / q n} & 0 \\
0 & e^{-\pi i p / q n}
\end{array}\right)
$$

Define Möbius transformations $F$ and $D$ as follows.

$$
F=A^{1 / 2} F_{1}
$$

where $F_{1}$ is the elliptic element of order two, keeping invariant the disc: $\{z \in \mathbf{C} ;|z|<1 / \lambda\}$, and with isometric circle $\mathcal{N}$ centered in a point on the ray $\{\arg (w)=0\}$ and tangent to the ray $\{a r g(w)=\pi / 3 n\}$. The circle $\mathcal{N}$ is necessarily orthogonal to the boundary of the above disc: The isometric: circles of $F$ and $F^{-1}$ are $\mathcal{N}$ and $A^{1 / 2}(\mathcal{N})$, respectively.

$$
D=A^{1 / 2} D_{1}
$$

where $D_{1}$ is the elliptic element of order two, keeping invariant the mnit disc, and with isometric circle $\mathcal{M}$ (necessarily orthogonal to the unit circle) with center on the ray $\{\arg (w)=0\}$ and tangent to the ray $\{\arg g(w)=$ $\pi / 3 n\}$. The isometric circles of $D$ and $D^{-1}$ are $\mathcal{M}$ and $A^{1 / 2}(\mathcal{M})$, respec:tively.

We assume $\lambda$ big enough such that the isometric circles of $F$ and $D$ are disjoint.

Denote by $L_{1}, L_{2}, L_{3}$ and $L_{4}$ the sets $\{\arg (w)=\pi / n\},\{\arg (w)=$ $-\pi / n\},\{|w|=1\}$ and $\left\{|w|=\lambda^{-1}\right\}$, respectively. If $M$ is a Möbius transformation, then $I(M)$ will denote its isometric circle. 
10.3. Some regions on the Riemann sphere. We proceed to define open regions in the complex plane, when $n$ is a prime, as follow's. The caste $n$ not a prime will be done in a later subsection.

The region $W_{k} \quad k \geq 1$ Set $\theta=\pi / n$ and $\mathcal{K}_{n}=\{z \in \mathbf{C} ;-\theta<a r g(z)<\theta\}$. We proceed firstly to the construction of some circles $C_{2} \ldots . ., C_{k}$, in case that $k>1$, so that:

(1) $C_{i}$ is orthogonal to $L_{3}$.

(2) $C_{i}$ is contained in $\mathcal{K}_{n}$.

(3) If $a_{i}$ denotes the center of the circle $C_{i}$, then $0<\arg \left(a_{2}\right)<\arg \left(a_{3}\right)<$ $\cdots<\arg \left(a_{k}\right)<\theta$.

(4) $C_{i}^{\prime}$ only intersects $C_{i+1}^{r}$, and they do it in an angle of $\theta$.

(5) If $\sigma_{i}$ denotes the reflection on $C_{i}$, and $\sigma_{0}(z)=\bar{z}$, then $\sigma_{i}\left(C_{i+1}\right) \cap$ $\sigma_{i}\left(\sigma_{i-1}\left(C_{i}\right)\right)=\emptyset$.

Set $\tilde{C}_{i}=\sigma_{i-1}\left(C_{i}\right), D_{i}$ the bounded disc: by $C_{i}^{\prime}$, and $\tilde{D}_{1}$ the bounded disc by $\tilde{C}_{1}$.

Set $W_{k}=\mathcal{K}_{n} \cap\left(\cup_{i=2}^{k} D_{i} \cup \tilde{D}_{1}\right)^{c}$. Denote also by $T_{i}=\sigma_{i} \sigma_{i-1}$.

The region $W_{k}$ is a fundamental set for a group $H_{k}$ with generators $A, B$, $T_{2}, \ldots, T_{k}$, and relations $A^{n}=B^{2}=(B A)^{2}=1, T_{i}^{n}=1,\left(T_{i} T_{i-1} \cdots T_{3} T_{2} B\right)^{2}=$ 1. The Riemamn surface $\Omega\left(H_{k}\right) / H_{k}$ has signature $(0,2+k ; 2,2, n, \ldots, n)$. The group $H_{k}$ is constructible from $H_{k-1}$ and $T_{k}$ by the second Maskit-Klem combination theorem (see [12]). Since $H_{1}$ is a finite group (dihedral group of order $2 n$ ), it is geometrically finite and has connected region of discontinuity. As a consequence, $H_{k}$ is a geometrically finite function group with connected region of discontinuity. In figure 5 we sketch the regions $W_{1}, W_{2}$ 
and $W_{3}$.
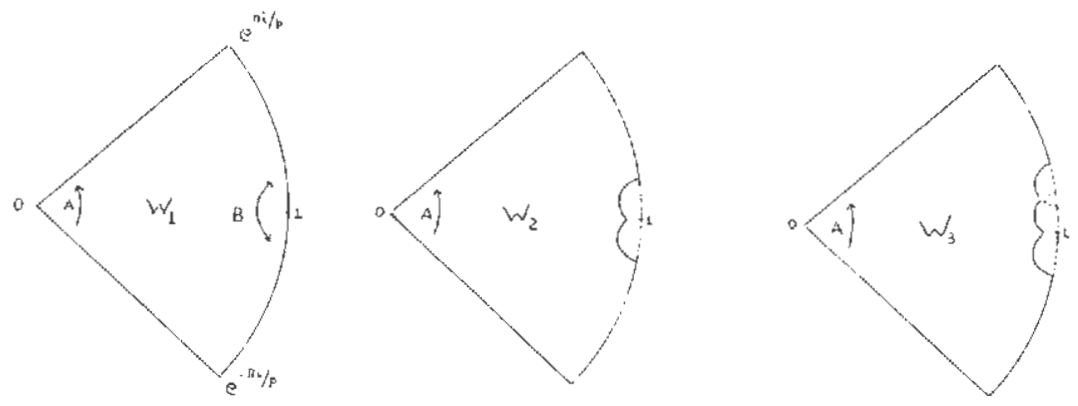

figure 5 
The region $R_{1}$. We define the region $R_{1}$ as the open region bonndert by $L_{1}, L_{2}, L_{3}$ and $L_{4}$.

The region $R_{2}$. We define the region $R_{2}$ as the open region bounded by $L_{1}, L_{2}, L_{3}, L_{4}, \mathrm{I}(D), \mathrm{I}\left(D^{-1}\right)$ and $\mathrm{I}\left(A^{-1} D^{-1} A\right)$.

The region $R_{3}$. We define the region $R_{3}$ as the open region bonnded by $L_{1}, L_{2}, L_{3}, L_{4}, \mathrm{I}(F), \mathrm{I}\left(F^{-1}\right), \mathrm{I}\left(A^{1} F^{-1} A\right), \mathrm{I}(D), \mathrm{I}\left(D^{-1}\right)$ and $\mathrm{I}\left(A^{-1} D^{1} A\right)$.

The regions $R_{1}, R_{2}$ and $R_{3}$ are drawn in figure 6 .

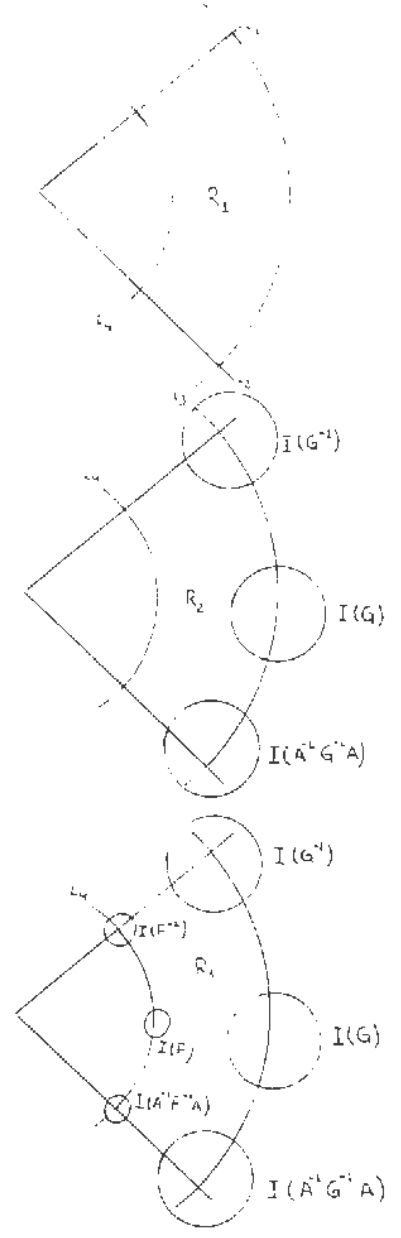

figure 6 
Another notations we will use, are the following.

(1) If $H$ is a subset of a group $K$, then we denote by $\ll H \gg$ the smallest normal subgroup of $K$ containing $H$.

(2) If $a_{1}, \ldots, a_{n}$ are elements of a group $K$, then we denote by $\left\langle a_{1}, \ldots, a_{n}\right\rangle$ the subgroup generated by these elements.

10.4. The constructions for $n$ prime. In this part, we proceed to construct the geometrically finite Kleinian groups in the case $n$ is a prime. First we use Maskit-Klein combination theorems (see [12]) to obtain a geometrically finite Kleinian group $J$ with connected region of discontimuity $\Omega$. Then we consider a nomal subgroup $G$ of $J$ such that $J / G$ is a dihedral group of order $2 n$. The group $G$ is a purely loxodromic, geometrically finite, function group with connected region of discontinuity. As a consequence of Maskit's classification of function groups (see [14]), we obtain that $G$ is in fact a Schottky group. The Riemann surface $\Omega / G$ is then a closed Riemann surface admitting a dihedral group of conformal automorphisms which lifts under the natural Schottky covering induced by $G$.

Once we have constructed a geometrically finite Kleimian group $J$ as desired, the theory of quasi-conformal maps, developed by L. Bers (see section 2), permits us to have a family of geometrically finite Kleinian groups as desired. This is done as follows: for each Beltrami coefficient $\mu$ for $J$ (see section 2), we consider the the $\mu$-quasiconformal homeonorphism $W_{\mu}: \hat{\mathbf{C}} \rightarrow \hat{\mathbf{C}}$ fixing the points 0,1 and $\infty$. Then Lemma 2.2 asserts that $J_{\mu}=W_{\mu} J W_{\mu}^{-1}$ is again a Kleinian group. In fact, $J_{\mu}$ is again a geometrically finite Kleinian group, which contains the Schottky group $G_{\mu}=W_{\mu} G W_{\mu}^{-1}$, with $J_{\mu} / G_{\mu}$ isomorphic to $D_{n}$.

Example (1). Signature $(0,2 r ; 2, \ldots, 2), r \geq 2$.

Let us consider the region $R_{1}$. Let $L_{1}, \ldots, L_{r-2}$, be elliptic elements of order two such that their isometric circles are contained in the region $R_{1}$, and they are pairwise disjoint. Let us consider the group (constructed from Maskit-Klein combination theorems)

$$
J=<A, B, C, L_{1}, \ldots, L_{r-2}>\text {. }
$$

As a consequence of Maskit-Klein combination theorems, the group $J$ is a geometrically finite function group with region of discontinuity $\Omega$ connected. The elements of $J$ are either loxodromics or conjugated to some power of a generator. The Riemann surface $\Omega / J$ is a closed Riemam surface of signature $(0,2 r ; 2, \ldots, 2), r \geq 2$. The normal subgroup 


$$
G=\ll A^{y} B^{t} C, A^{y_{1}} B L_{1}, \ldots, A^{s_{r-2}} B L_{r} \cdot 2 》,
$$

where $s, s_{j} \in\{0,1, \ldots, n-1\}$ and $t \in\{0,1\}$, is a purely loxodromic: geometrically finite function group with comnected region of discontinuty. As a consequence, $G$ is a Schottky group (see [14]). Moreover, ( $G$ lhas index $2 n$ and $J / G$ is a dihedral group of order $2 p$.

Example (2) Signature $(0,2 r+k ; 2, \ldots, 2, n, \ldots, n), k \geq 1$ and $r \geq 1$. Let us consider the region $W_{k}$, and the elliptic transformations $T_{2} \ldots, T_{k}$. Let $L_{1}, \ldots, L_{r-1}$ be elliptic elements of order two sur th that their isometric: circles are contained in the region $W_{k}$ and they are pairwise disjoint.

Let us consider the group

$$
J=<A, B, L_{1}, \ldots, L_{r-1}, T_{2}, \ldots, T_{k}>.
$$

Same arguments as before show that the group $J$ is a geometrically finite function group with region of discontinuity $\Omega$ connected. The elements of $J$ are either loxodromics or conjugated to some power of a generator. The Riemann surface $\Omega / J$ is a closed Riemamn surface of signature $(0,2 r+$ $k ; 2, \ldots, 2, n, \ldots, n), k \geq 1$ and $r \geq 1$. The normal subgroup

$$
G=\ll A^{s_{1}} B L_{1}, \ldots, A^{s_{T-1}} B L_{r-1}, A^{t_{2}} T_{2}, \ldots, A^{t_{k}} T_{k} \gg, s \in\{1,-1\},
$$

where $t_{i} \in\{1, \ldots, n-1\}$ and $s_{j} \in\{0,1, \ldots, n-1\}$, is a purely loxodromic: geometrically finite function group with connected region of discontinuity. As a consequence, the group $G$ is a Schottky group (see [14]). Moreover, $G$ has index $2 n$ and $J / G$ is dihedral group.

Example (3) Signature $(1,2 r ; 2, \ldots, 2), r \geq 1$.

Let us consider the region $R_{2}$. Let $L_{1}, \ldots, L_{r-1}$ be elliptic elements of order two such that their isometric circles are contained in the region $R_{2}$ and they are pairwise disjoint. Let us consider the geometrically finite function group with connected region of discontinuity, given by

$$
J=<A, B, C, D, L_{1}, \ldots, L_{r-1}>.
$$

If $\Omega$ denotes the region of discontinuity of $J$, then the Riemann surface $\Omega / J$ is a closed Riemann surface of signature $(1,2 r ; 2, \ldots, 2), r \geq 1$. Let us consider the normal subgroup

$$
G=\ll A^{s} B^{t} C, A^{u} B^{v} D, A^{s_{1}} B L_{1}, \ldots, A^{s_{r-1}} B L_{r-1} \gg,
$$

where $u, s, s_{j} \in\{0,1, \ldots, n-1\}$ and $t, v \in\{0,1\}$. As before, $C^{\prime} \mathrm{r}$ is a Schottky group such that $J / G$ is dihedral of order $2 n$. 
Example (4) Signature $(1,2 r+k ; 2, \ldots, 2, n, \ldots, n), k \geq 1$ and $r \geq 0$.

Let us consider the region $W_{k}$ and the transformations $T_{2}, \ldots, T_{k}$. Let $L_{1}, \ldots, L_{r}$ be elliptic (if $r \geq 1$ ) elements of order two such that their isometric: circles are contained in the region $W_{k}$ and they are pairwise disjoint. We consider a loxodromic transformation $E$ which keeps invariant the unit dis: and conjugate $T_{k} \cdots T_{2} B$ to $A B$. We may assume the multiplier big enongh so we can apply the second Maskit-Klein combination theorem to the group $<H_{k}, L_{1}, \ldots, L_{r}>$ and $E$. Let us consider the geometrically finite function group with connected region of discontinuity, given by

$$
J=<A, B, E, L_{1}, \ldots, L_{r}, T_{2}, \ldots, T_{k}>.
$$

If $\Omega$ denotes the region of discontinuity of $J$, then the Riemann surface $\Omega / J$ is a closed Riemann surface of signature $(1,2 r+k ; 2, \ldots, 2, n, \ldots, n)$, $k \geq 1$ even and $r \geq 0$. Let us consider the normal subgroup

$$
G=\ll A^{s} B^{t} E, A^{s_{1}} B L_{1}, \ldots, A^{s_{r}} B L_{r}, A^{t_{2}} T_{2}, \ldots, A^{t_{k}} T_{k} \gg,
$$

where $s, s_{j} \in\{0,1, \ldots, n-1\}, t_{i} \in\{1, \ldots, n-1\}$ and $t \in\{0,1\}$. The group $G$ is a Schottky group, and $J / G$ is dihedral of order $2 n$.

Example (5) $(\gamma, 2 r ; 2, \ldots, 2), \gamma \geq 2$ and $r \geq 2$.

Let us consider the region $R_{1}$. Let $L_{1}, \ldots, L_{r-2}$ be elliptic elements of order two, and $A_{t}, t=1, \ldots, \gamma$, be loxodromic elements with $I\left(A_{i}\right) \cap I\left(A_{i}^{m}\right)=$ $\emptyset$ all $i=1, \ldots, \gamma$, and $m \in\{-1,+1\}$. Assume all the isometric circles $I\left(L_{j}\right)$, $I\left(A_{i}\right), I\left(A_{l}^{-1}\right)$ in the region $R_{1}$ and pairwise disjoint. Let us consider the geometrically finite function group with connected region of discontinuity, given by

$$
J=<A, B, C, A_{1}, \ldots, A_{\gamma}, L_{1}, \ldots, L_{r-2}>.
$$

If $\Omega$ denotes the region of discontinuity of $J$, then the Riemann surface $\Omega / J$ is a closed Riemann surface of signature $(\gamma, 2 r ; 2, \ldots, 2), \gamma \geq 2$ and $r \geq 2$. Let us consider the normal subgroup

$$
G=\ll A^{t} B^{u} C, A^{d_{1}} B^{\epsilon_{1}} A_{1}, \ldots, A^{d_{\gamma}} B^{\epsilon_{\gamma}} A_{\gamma}, A^{s_{1}} B L_{1}, \ldots, A^{s_{r-2}} B L_{r-2} \gg,
$$

where $t, s_{j}, d_{i} \in\{0,1, \ldots, n-1\}, u, e_{j} \in\{0,1\}$. The group $G$ is a Schot tky group with $J / G$ a dihedral group of order $2 n$. 
Example (6) $(\gamma, 2 r ; 2, \ldots, 2), \gamma \geq 2$ and $r \geq 1$.

Let us consider the region $R_{3}$. Let $L_{1}, \ldots, L_{r}$ be elliptic elements of order two, and $A_{t}, t=1, \ldots, \gamma-2$, be loxodromic elements such that $I\left(A_{i}\right) \cap I\left(A_{j}^{m}\right)=\emptyset$, where $m \in\{-1,+1\}$, the isometric circles $I\left(L_{i}\right), I\left(A_{j}^{m}\right)$ are contained in the region $R_{3}$ and they are all pairwise disjoint. Let us consider the geometrically finite function group with comnected region of discontinuity, given by

$$
J=<A, B, C, F, D, A_{1}, \ldots, A_{\gamma-2}, L_{1}, \ldots, L_{r}>.
$$

If $\Omega$ denotes the region of discontinuity of $J$, then the Riemann surface $\Omega / J$ is a closed Riemann surface of signature $(\gamma, 2 r ; 2, \ldots, 2), \gamma \geq 2$ and $r \geq 1$. Let us consider the normal subgroup

$$
\begin{aligned}
G=\ll & A^{s_{1}} B^{t_{1}} C, A^{s_{2}} B^{t_{2}} F, A^{s_{3}} B^{t_{3}} D, A^{d_{1}} B^{e_{1}} A_{1}, \ldots, \\
& , A^{d_{\gamma-2}} B^{\epsilon_{\gamma-2}} A_{\gamma-2}, A^{h_{1}} B L_{1}, \ldots, A^{h_{r}} B L_{r} \gg
\end{aligned}
$$

where $s_{i}, d_{j}, h_{m} \in\{0,1, \ldots, n-1\}$ and $t_{i}, e_{j} \in\{0,1\}$. The group $G$ is a Schottky group and $J / G$ is dihedral of order $2 n$.

Example (7) $(\gamma, 2 r ; 2, \ldots, 2), \gamma \geq 2$ and $r \geq 1$.

Let us consider the region $R_{2}$. Let $L_{1}, \ldots, L_{r-1}$ be elliptic elements of order two, and $A_{t}, t=1, \ldots, \gamma-1$, be loxodromic elements such that $I\left(A_{i}\right) \cap I\left(A_{j}^{m}\right)=\emptyset$, where $m \in\{-1,+1\}$. Assume all the isometric circles in the region $R_{2}$ and pairwise disjoint. Let us consider the geometrically finite function group with connected, region of discontinuity, given by

$$
J=<A, B, C, D, A_{1}, \ldots, A_{\gamma-1}, L_{1}, \ldots, L_{r-1}>.
$$

If $\Omega$ denotes the region of discontinuity of $J$, then the Riemamn surface $\Omega / J$ is a closed Riemann surface of signature $(\gamma, 2 r ; 2, \ldots, 2), \gamma \geq 2$ and $r \geq 1$. Let us consider the normal subgroup

$$
\begin{aligned}
G=\ll & A^{s} B^{u} C, A^{v} B^{t} D, A^{d_{1}} B^{\epsilon_{1}} A_{1}, \ldots, A^{d_{\gamma-1}} B^{\epsilon_{\gamma-1}} A_{\gamma-1}, A^{s_{1}} B L_{1}, \ldots, \\
& , A^{s_{r-1}} B L_{r-1} \gg,
\end{aligned}
$$

where $s, v, d_{i}, s_{j} \in\{0,1, \ldots, n-1\}$ and $u, t, e_{i} \in\{0,1\}$. The group $G$ is a Schottky group with $J / G$ dihedral of order $2 n$. 
Example (8) $(\gamma, 2 r+k ; 2, \ldots, 2, n, \ldots, n), \gamma \geq 2, k \geq 1$ and $r \geq 1$.

Let us consider the region $W_{k}$ and the transformations $T_{2}, \ldots, T_{k}$. Let $L_{1}, \ldots, L_{r-1}$ be elliptic elements of order two, and $A_{t}, t=1, \ldots, \gamma$, be loxodromic elements with $I\left(A_{i}\right) \cap I\left(A_{i}^{m}\right)=\emptyset$ all $i=1, \ldots, \gamma$ and $m \in\{-1,+1\}$. Assume all the isometric circles $I\left(L_{j}\right), I\left(A_{i}\right), I\left(A_{l}^{-1}\right)$ in the region $W_{k}$ and pairwise disjoint. Let us consider the geometrically finite function group with connected region of discontimuity, given by

$$
J=<A, B, A_{1}, \ldots, A_{\gamma}, L_{1}, \ldots, L_{r-1}, T_{2}, \ldots, T_{k}>.
$$

If $\Omega$ denotes the region of discontinuity of $J$, then the Riemann surface $\Omega / J$ is a closed Riemann surface of signature

$$
(\gamma, 2 r+k ; 2, \ldots, 2, n, \ldots, n)
$$

$\gamma \geq 2, k \geq 1$ and $r \geq 1$. Let us consider the normal subgroup

$$
G=\ll
$$

$A^{d_{1}} B^{e_{1}} A_{1}, \ldots, A^{d_{\gamma}} B^{\epsilon_{\gamma}} A_{\gamma}, A^{s_{1}} B L_{1}, \ldots, A^{s_{r-1}} B L_{r-1}, A^{t_{2}} T_{2}, \ldots, A^{t_{k}} T_{k} \gg$,

where $d_{i}, s_{j} \in\{0,1, \ldots, n-1\}, e_{i} \in\{0,1\}$ and $t_{j} \in\{1, \ldots, n-1\}$. The group $G$ is a Schottky group with $J / G$ a dihedral of order $2 n$.

Example (9) $(\gamma, 2 r+k ; 2, \ldots, 2, n, \ldots, n), \gamma \geq 2, k \geq 1$ and $r \geq 0$.

Let us consider the region $W_{k}$ and the transformations $T_{2}, \ldots, T_{k}$. Let $L_{1}, \ldots, L_{r}$ be elliptic elements of order two, and let $A_{t}, t=1, \ldots, \gamma-1$, be loxodromic elements such that $I\left(A_{i}\right) \cap I\left(A_{j}^{m}\right)=\emptyset$, where $m \in\{-1,+1\}$. Assume all the isometric circles $I\left(L_{i}\right), I\left(A_{j}^{m}\right)$ in the region $W_{k}$ and pairwise disjoint. Let us consider a transformation $E$ as in example (4). Let us consider the geometrically finite function group with connected region of discontinuity, given by

$$
J=<A, B, E, A_{1}, \ldots, A_{\gamma-1}, L_{1}, \ldots, L_{r}, T_{2}, \ldots, T_{k}>.
$$

If $\Omega$ denotes the region of discontinuity of $J$, then the Riemann surface $\Omega / J$ is a closed Riemann surface of signature

$$
(\gamma, 2 r+k ; 2, \ldots, 2, n, \ldots, n)
$$

$\gamma \geq 2, k \geq 1$ and $r \geq 0$. Let us consider the normal subgroup

$G=\ll A^{s} B^{u} E, A^{d_{1}} B^{e_{1}} A_{1}, \ldots, A^{d_{\gamma-1}} B^{e_{\gamma-1}} A_{\gamma-1}, A^{s_{1}} B L_{1}, \ldots, A^{s_{r}} B L_{r}, A^{t_{2}} T_{2}, \ldots$, , $A^{t_{k}} T_{k} \gg$,

where $s, d_{i}, s_{j} \in\{0,1, \ldots, n-1\}, u, e_{i} \in\{0,1\}$ and $t_{j} \in\{1, \ldots, n-1\}$. The group $G$ is a Schottky group with $J / G$ a dihedral group of order $2 n$. 
Example (10) $(\gamma, 2 r+k ; 2, \ldots, 2, n, \ldots, n), \gamma \geq 2, k \geq 1$ and $r \geq 0$.

Let us consider the region $W_{k}$ and the transformations $T_{2}, \ldots, T_{k}$. Let $L_{1}, \ldots, L_{r}$ be elliptic elements of order two, and $A_{t}, t=1, \ldots, \gamma-1$. be loxodromic elements such that $I\left(A_{i}\right) \cap I\left(A_{j}^{m}\right)=\emptyset$, where $m \in\{-1 .+1\}$. Assume all the isometric circles in the region $W_{k}$ and pairwise disjoint. Let $E$ be a transformation as in example (4). Let us consider the geometric:ally finite function group with connected region of discontinuity, given by

$$
J=<A, B, E, A_{1}, \ldots, A_{\gamma-1}, L_{1}, \ldots, L_{r}, T_{2}, \ldots . T_{k}>.
$$

If $\Omega$ denotes the region of discontinuity of $J$, then the Riemann surface $\Omega / J$ is a closed Riemann surface of signature

$$
(\gamma, 2 r+k ; 2, \ldots, 2, n, \ldots, n)
$$

, $\gamma \geq 2, k \geq 2$ even and $r \geq 0$. Let us consider the normal subgroup

$$
\begin{aligned}
G=\ll & A^{s} B^{t} E, A^{d_{1}} B^{\epsilon_{1}} A_{1} \ldots, A^{d_{\gamma-1}} B^{e_{\gamma-1}} A_{\gamma-1}, A^{s_{1}} B L_{1}, \ldots, A^{s_{r}} B L_{r}, A^{t_{2}} T_{2}, \ldots, \\
& , A^{t_{k}} T_{k} \gg
\end{aligned}
$$

where $s, d_{i}, s_{j} \in\{0,1, \ldots, n-1\}, t, e_{i} \in\{0,1\}$ and $t_{j} \in\{1, \ldots, n-1\}$. The group $G$ is a Schottky group with $J / G$ a dihedral of order $2 n$.

Example (11) $(\gamma, 0 ;-), \gamma \geq 2$.

Let us consider the region $R_{3}$. Let $A_{1}, \ldots, A_{\gamma-2}$, be loxodromic elements such that $I\left(A_{i}\right) \cap I\left(A_{j}^{m}\right)=\emptyset$, where $m \in\{-1,+1\}$, and all these isometric; circles are contained in the region $R_{3}$. Let us consider the geometrically finite function group with connected region of discontinuity, given by

$$
J=<A, B, C, F, D, A_{1}, \ldots, A_{\gamma-2}>.
$$

If $\Omega$ denotes the region of discontinuity of $J$, then the Riemamn surface $\Omega / J$ is a closed Riemann surface of signature $(\gamma, 0 ;-), \gamma \geq 2$. Let us consider the normal subgroup

$$
G=\ll A^{s_{1}} B^{t_{1}} C, A^{s_{2}} B^{t_{2}} F, A^{s_{3}} B^{t_{3}} D, A^{d_{1}} B^{\varepsilon_{1}} A_{1}, \ldots, A^{d_{\gamma-2}} B^{\epsilon_{\gamma-2}} A_{\gamma-2} \gg,
$$

where $s_{i}, d_{j} \in\{0,1, \ldots, n-1\}$ and $t_{i}, e_{j} \in\{0,1\}$. The group $G$ is a Schottky group and $J / G$ is dihedral of order $2 n$. 
10.5. The construction in the general case. The above examples can be carry out for any integer $n \geq 3$. For this we only need to consider new regions $W_{k}^{1}\left(d_{2}, \ldots, d_{k}\right), W_{k}^{2}\left(d_{2}, \ldots, d_{k}\right)$ and $\mathbb{W}_{k}^{3}\left(d_{2}, \ldots, d_{k}\right)$, where $1<d_{2}<d_{3}<\cdots<d_{k}<n, d_{i}$ dividing $n$. The region $\|_{k}^{1}\left(d_{2} \ldots . d_{k}\right)$ is defined in analogous way as the region $W_{k}$, but in this case the transformations $T_{j}$ in the construction may have order $n / d_{j}$. The region $I t_{k}^{-2}\left(d_{2}, \ldots . d_{k}\right)$ is constructed from region $W_{k}^{1}\left(d_{2}, \ldots, d_{k}\right)$ but deleting the disc: of radins $1 / \lambda$ and center 0 (in this construction, we are assuming that $\lambda$ is big enongh so) that the circle of radius $1 / \lambda$ does not cut the boundary of $W_{k}^{1}\left(d_{2}, \ldots d_{k}\right)$ ). The region $W_{k}^{3}\left(d_{2}, \ldots, d_{k}\right)$ is constructed from the region ${ }^{2}{ }_{k}^{2}\left(d_{2} \ldots . . d_{k}\right)$ by deleting the bounded discs with boundary the isometric circles $I(F)$, $I\left(F^{-1}\right)$ and $I\left(A^{-1} F^{-1} A\right)$, respectively (again the value of $\lambda$ is assumed big enough so that the circles $I(F), I\left(F^{-1}\right)$ and $I\left(A^{-1} F^{-1} A\right)$ do not cut the boundary of $\left.W_{k}^{2}\left(d_{2}, \ldots, d_{k}\right)\right)$. We have $W_{k}^{1}(1, \ldots, 1)=W_{k}$. As a consequence of propositions 6.2 and 6.3, we get all topological actions of such a group. We proceed to give an example for $n=4$ (to simplify).

Example $n=4$ and $\gamma=0$ 。 In this example, $H$ is generated by elements $a$ and $b$ of order 4 and 2 , respectively. The values $r_{1}$ and $r_{2}$ are the number of fixed points of $b$ and $a b$, respectively. The numbers $k_{1}$ and $k_{2}$ are the number of fixed points of $a$ and $a^{2}$ (which are not fixed by $a$ ), respectively.

The signature $\left(0, r_{1}+r_{2} ; 2, \ldots, 2\right)$ with $r_{1}$ and $r_{2}$ even Let us consider the region $R_{1}$ and elliptic transformation of order two, say $L_{1}, \ldots$, $L_{\left(r_{1}-2\right) / 2}, N_{1}, \ldots, N_{\left(r_{2}-2\right) / 2}$, so that their isometric circles are contained in the region $R_{1}$ and they are pairwise disjoint. Let $J$ be the group generated by $A, B, C, L_{1}, \ldots, L_{\left(r_{1}-2\right) / 2}, N_{1}, \ldots, N_{\left(r_{2}-2\right) / 2}$. As a consequence of MaskitKlein combination theorems, we have that $J$ is a geometrically finite func:tion group with connected region of discontinuity and every non-loxodromic: element is conjugate to a power of a generator. Let $G$ be the smallest normal

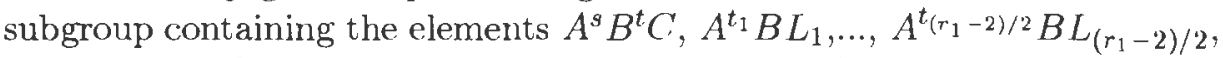

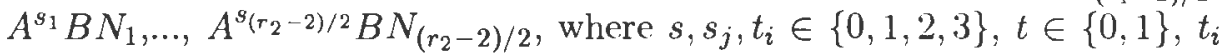
even and $s_{j}$ odd. The group $G$ happen to be a purely loxodromic, geormetrically finite function group with connected region of discontinuity. It follows that $G$ is a Schottky group of index 8 in $J$.

The signature $\left(0, r_{1}+r_{2} ; 2, \ldots, 2\right)$ with $r_{1}$ and $r_{2}$ odd Let us consider the region $R_{2}$ and elliptic transformation of order two, say $L_{1}, \ldots$, $L_{\left(r_{1}-1\right) / 2}, N_{1}, \ldots, N_{\left(r_{2}-1\right) / 2}$, so that their isometric circles are contained in the region $R_{2}$ and they are pairwise disjoint. Let $J$ be the group gen- 
erated by $A, B, C, D, L_{1}, \ldots, L_{\left(r_{1}-1\right) / 2}, N_{1}, \ldots, N_{\left(r_{2}-1\right) / 2}$. As a consequence of Maskit-Klein combination theorems, we have that $J$ is a geometrically finite function group with connected region of discontinuity and every non-loxodromic element is conjugate to a power of a generator. Let $G$ be the smallest normal subgroup containing the elements $A^{s} B^{t} C$,

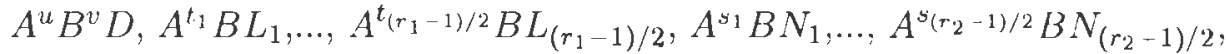
where $u, s, s_{j}, t_{i} \in\{0,1,2,3\}, t, v \in\{0,1\}, t_{i}$ cven and $s_{j}$ odd. The group $G$ happen to be a purely loxodromic, geometrically finite function group with comnected region of discontinuity. It follows that $(x$ is a Schottky group of index 8 in $J$.

The signature $\left(0, k_{1}+k_{2}+r_{1}+r_{2} ; 2, \ldots, 2,4, \ldots, 4,2, \ldots, 2\right)$ with $r_{1}$ and $r_{2}$ even and $k_{1}>0$ Let us consider the region $W_{k_{1}+k_{2}}^{1}(1, \ldots, 1,2, \ldots, 2)$, where the number 1 is $k_{1}-1$ times and the number 2 is $k_{2}$ times. We also consider the transformations $T_{2}, \ldots, T_{k_{1}+k_{2}}$ in the construction of such a region. Let us also consider elliptic transformations of order two, say $L_{1}, \ldots, L_{r_{1} / 2}, N_{1}, \ldots, N_{r_{2} / 2}$, so that their isometric circles are contained in the above region, and they are pairwise disjoint. Let $E$ be a loxodromic; transformation which conjugate $T_{k_{1}+k_{2}} \cdots T_{2} B$ onto $A B$. We choose it with big multiplier so that we can apply the second Maskit-Klein combination theorem to the group generated by the above transformations. Let $J$ be the group generated by $A, E, T_{2}, \ldots, T_{k_{1}+k_{2}}, L_{1}, \ldots, L_{r_{1} / 2}, N_{1}, \ldots, N_{r_{2} / 2}$. As a consequence of Maskit-Klein combination theorems, we have that $J$ is a geometrically finite function group with connected region of discontinuity and every non-loxodromic element is conjugate to a power of a generator. Let $G$ be the smallest normal subgroup containing the elements $A^{s} B^{t} E$, $A^{n_{2}} T_{2}, \ldots, A^{n_{k_{1}}} T_{k_{1}}, A^{2} T_{k_{1}+1}, \ldots A^{2} T_{k_{1}+k_{2}}, A^{t_{1}} B L_{1}, \ldots, A^{t_{\left(r_{1}-1\right) / 2} B L_{\left(r_{1}-1\right) / 2},}$

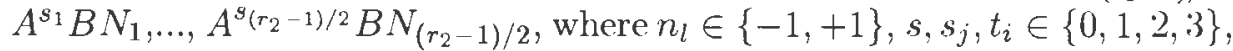
$t \in\{0,1\}, t_{i}$ evern and $s_{j}$ odd. The group $G$ happen to be a purely loxodromic, geometrically finite function group with connected region of discontinuity. It follows that $G$ is a Schottky group of index $8 \mathrm{in} J$.

The signature $\left(0, k_{1}+k_{2}+r_{1}+r_{2} ; 2, \ldots, 2,4, \ldots, 4,2, \ldots, 2\right)$ with $r_{1}$ and $r_{2}$ odd and $k_{1}>0$ Let us consider the region $W_{k_{1}+k_{2}}^{1}(1, \ldots, 1,2, \ldots, 2)$, where the number 1 is $k_{1}-1$ times and the number 2 is $k_{2}$ times. We also consider the transformations $T_{2}, \ldots, T_{k_{1}+k_{2}}$ in the construction of such a region. Let us also consider elliptic transformations of order two, say $L_{1}, \ldots, L_{\left(r_{1}-1\right) / 2}, N_{1}, \ldots, N_{\left(r_{2}-1\right) / 2}$, so that their isometric circles are contained in the above region, and they are pairwise disjoint. Let $J$ be the group generated by $A, T_{2}, \ldots, T_{k_{1}+k_{2}}, L_{1}, \ldots, L_{\left(r_{1}-1\right) / 2}, N_{1}, \ldots, N_{\left(r_{2}-1\right) / 2}$. As a consequence of Maskit-Klein combination theorems, we have that $J$ is 
a geometrically finite function group with connected region of discontinuity and every non-loxodromic element is conjugate to a power of a generator. Let $(r$ be the smallest normal subgroup containing the elements $A^{n_{2}} T_{2}, \ldots, A^{n_{k_{1}}} T_{k_{1}}, A^{2} T_{k_{1}+1}, \ldots \quad A^{2} T_{k_{1}+k_{2}}: A^{t_{1}} B L_{1}, \ldots, A^{t_{\left(r_{1}-1\right) / 2} B L_{\left(r_{1}-1\right) / 2}}$. $A^{s_{1}} B N_{1}, \ldots, A^{s\left(r_{2}-1\right) / 2} B N_{\left(r_{2}-1\right) / 2}$, where $n_{l} \in\{-1,+1\}, s_{j}, t_{i} \in\{0,1,2,3\}$. $t_{i}$ even and $s_{j}$ odd. The group $G$ happen to be a purely loxodromic; ger netrically finite function group with connested region of discontinnity. It follows that $C$ is a Schottky group of irklex 8 in $J$.

The signature $\left(0, k_{2}+r_{1}+r_{2} ; 2, \ldots, 2\right)$ with $r_{1}$ and $r_{2}$ even and $k_{2}>0$ Let us consider the region $W_{1+k_{2}}^{2}(2, \ldots, 2)$, where the number 2 is $k_{2}+r_{1}+r_{2}$ times. We also consider the transfornations $T_{2}, \ldots, T_{1+k_{2}}$ in the construction of such a region. Let us also consider elliptic transformations of order two, say $L_{1}, \ldots, L_{\left(r_{1}-2\right) / 2}, N_{1}, \ldots, N_{\left(r_{2}-2\right) / 2}$, so that their isometric circles are contained in the above region, and they are pairwise disjoint. Let $J$ be the group generated by $A, T_{2}, \ldots, T_{1+k_{2}}, L_{1}, \ldots, L_{\left(r_{1}-2\right) / 2}$, $N_{1}, \ldots, N_{\left(r_{2}-2\right) / 2}$. As a consequence of Maskit-Klein combination theorems, we have that $J$ is a geometrically finite function group with connected region of discontinuity and every non-loxodromic element is conjugate to a power of a generator. Let $G$ be the smallest normal subgroup containing

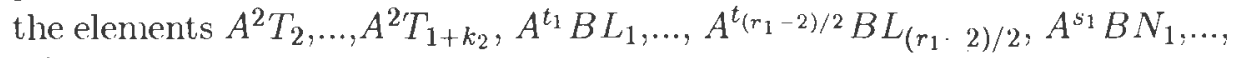
$A^{s\left(r_{2}-2\right) / 2} B N_{\left(r_{2}-2\right) / 2}$, where

$s_{j}, t_{i} \in\{0,1,2,3\}, t_{i}$ even and $s_{j}$ odd. The group $G$ happen to be a purely loxodronic; geometrically finite function group with connected region of discontinuity. It follows that $G$ is a Schottky group of index $8 \mathrm{in} J$.

The signature $\left(0, k_{2}+r_{1}+r_{2} ; 2, \ldots, 2\right)$ with $r_{1}$ and $r_{2}$ odd and $k_{2}>0$ Let us consider the region $W_{1+k_{2}}^{3}(2, \ldots, 2)$, where the number 2 is $k_{2}+r_{1}+r_{2}$ times. We also consider the transformations $T_{2}, \ldots, T_{1+k_{2}}$ in the construction of such a region. Let us also consider elliptic transformations of order two, say $L_{1}, \ldots, L_{\left(r_{1}-1\right) / 2}, N_{1}, \ldots, N_{\left(r_{2}-1\right) / 2}$, so that heir isometric: circles are contained in the above region, and they are pairwise disjoint. Let $J$ be the group generated by $A, F, T_{2}, \ldots, T_{1+k_{2}}, L_{1}, \ldots, L_{\left(r_{1}-1\right) / 2}, N_{1}, \ldots$, $N_{\left(r_{2}-1\right) / 2}$. As a consequence of Maskit-Klein combination theorems, we have that $J$ is a geometrically finite function group with connected region of discontinuity and every non-loxodromic element is conjugate to a power of a generator. Let $G$ be the smallest normal subgroup contain-

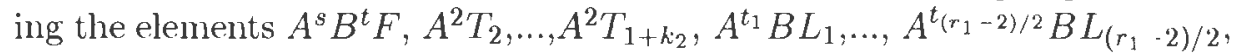

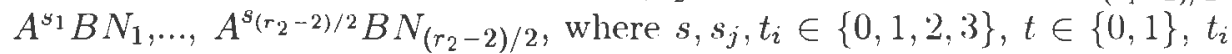
even and $s_{j}$ odd. The group $G$ happen to be a purely loxodronic, geometrically finite function group with connected region of discontinuity. It 
follows that $G$ is a Schottky group of index 8 in $J$.

\section{References}

[1] L. Bers. Uniformization by Beltrami equations. Comm. Pure Appl. Math. 14 , pp. 215-228, 1961.

[2] L. Bers. Automorphic forms for Schottky groups. Adv, in Math. 16, 1975 .

[3] H. Farkas and I. Kra Riemann surfaces. Springer-Verlag; New York, 1980.

[4] R.A. Hidalgo. On Schottky groups with automorphisms. Theses Ph.D. in Mathematics. S.U.N.Y. at Stony Brook, New York, (1991).

[5] R.A. Hidalgo. On $\gamma$-hyperelliptic Schottky groups. Notas Soc. Mat. de Chile 8 , pp. $27-36,1989$.

[6] R.A. Hidalgo. On Schottky groups with automorphisms. Ann. Acad. Fenn. $19, \mathrm{~N}^{\circ} .2$, pp. $259-289,1994$.

[7] R.A. Hidalgo. Schottky uniformizations of closed Riemann surfaces with the Klein group as conformal group of symmetries. Pre-print.

[8] R.A. Hidalgo. Schottky uniformizations with Abelian groups of conformal Automorphisms. Glasgow Math. J. 36 , pp. 17 - 32, 1994.

[9] R.A. Hidalgo. The Mixed Elliptically Fixed Point property for Kleinian groups. Ann. Acad. Fenn. 19, No.2, pp. $247-258,1994$.

[10] L. Keen. On hyperelliptic Schottky groups. Ann. Acad. Sci. Fenn.. series A.I. Mathematica 5, 1980.

[11] S. Kerckhoff. The Nielsen realization problem. Annals of Math. 117 , pp. 235 - 265, 1983.

[12] B. Maskit. Kleinian groups. Grundlehren der Mathematischen Wissenschaften, Vol. 287,Springer - Verlag, Berlin, Heildelberg, New York, 1988.

[13] B. Maskit. A characterization of Schottky groups. J. d'Analyse Math. 19 , pp. $227-230,1967$. 
[14] B. Maskit. On the classification of Kleinian groups I and II. Acta Math. 135 and 138, 1975, 1977.

[15] B. Maskit, Canonical domains on Riemann surfaces, Proc. of the Amer. Math. Soc. 106), pp. $713-721,1989$.

[16] J. Morgan. On Thurston's uniformization theorem for threedimensional manifolds. The Smith Conjecture (H. Bass and J. Morgan, eds), Academic Press, San Diego, 1984.

[17] C. O'ryan. Unifpack: Un paquete para calcular uniformizaciones de superficies de Riemann con automorfismos. Thesis Licenciatura en matemáticas, Pontificia Universidad Católica de Chile, Santiago, Chile, 1992.

Received : November 10, 1995

\section{Rubén A. Hidalgo}

Departamento de Matemáticas

Universidad Técnica Federico Santa María

Casilla 110-V

Valparaíso, Chile. 